Tom Fisher

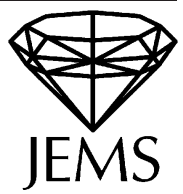

\title{
Some examples of 5 and 7 descent for elliptic curves over $\mathbf{Q}$
}

Received October 6, 2000 / final version received November 14, 2000

Published online February 15, 2001 - (C) Springer-Verlag \& EMS 2001

\begin{abstract}
We perform descent calculations for the families of elliptic curves over $\mathbf{Q}$ with a rational point of order $n=5$ or 7 . These calculations give an estimate for the MordellWeil rank which we relate to the parity conjecture. We exhibit explicit elements of the Tate-Shafarevich group of order 5 and 7, and show that the 5-torsion of the Tate-Shafarevich group of an elliptic curve over $\mathbf{Q}$ may become arbitrarily large.
\end{abstract}

\section{Introduction}

Let $E$ be an elliptic curve over a number field $K$. The Mordell-Weil theorem asserts that the group $E(K)$ of $K$-rational points on $E$ is a finitely generated abelian group. We call $E(K)$ the Mordell-Weil group. Another important group associated to $E$ is the Tate-Shafarevich group $\amalg(E / K)$. It is defined as the set of torsors under $E$ which have points everywhere locally. Thus a non-zero element of the Tate-Shafarevich group corresponds to a smooth curve of genus 1 that violates the Hasse Principle, i.e. it has (local) $K_{v}$-rational points at all places $v$, but no (global) $K$-rational points. It is known that $\amalg(E / K)$ is torsion, and that the torsor corresponding to an element of order $n$ admits a divisor class of degree $n$ defined over $K$. On a curve of genus 1 a complete linear system of degree $n$ also has dimension $n$. Thus for $n=2$ we consider double covers of $\mathbf{P}^{1}$, for $n=3$ plane cubics, for $n=4$ complete intersections of two quadrics in $\mathbf{P}^{3}$, and so on.

There is no known algorithm guaranteed to compute the Mordell-Weil group, and the situation for the Tate-Shafarevich group is no better. However, for any integer $n \geq 2$ the proof of the Mordell-Weil theorem gives an effective upper bound on the order of $E(K) / n E(K)$. This yields an upper bound on the Mordell-Weil rank which is an overestimate precisely whenever $\amalg(E / K)$ contains non-trivial $n$-torsion. Such calculations are referred to as descent calculations. By performing an $n$-descent we obtain partial information concerning the groups $E(K)$ and $\amalg(E / K)$. It is therefore of interest to make descent calculations practical for as many different values of $n$ as possible.

A great deal of work has been done on 2- and 3-descents, the advantage of $n$ being small outweighing the fact that the primes 2 and 3 often require special

T. Fisher: University of Cambridge, DPMMS, Centre for Mathematical Sciences, Wilberforce Road, Cambridge CB3 OWB, UK 
treatment in the study of elliptic curves. We give some examples of 5- and 7descents. Unfortunately we work in a special case, so our results do not apply to an arbitrary elliptic curve. Firstly, our descent calculations assume the existence of an isogeny of degree $n=5$ or 7 . Secondly, we assume one of our pair of elliptic curves has a rational point of order $n$. Notice that by a result of Serre [Se1], the second condition is automatically satisfied if we restrict to semi-stable elliptic curves. Here we recall a curve is semi-stable if it has good or multiplicative reduction at all primes.

Accordingly we consider pairs of elliptic curves $C$ and $D$ defined over $K$, related by the exact sequences of Galois modules

$$
0 \rightarrow \mu_{n} \rightarrow C \stackrel{\phi}{\rightarrow} D \rightarrow 0 \quad \text { and } \quad 0 \rightarrow \mathbf{Z} / n \mathbf{Z} \rightarrow D \stackrel{\widehat{\phi}}{\rightarrow} C \rightarrow 0
$$

In other words $\phi: C \rightarrow D$ is an isogeny of degree $n$ with kernel a labelled copy of $\mu_{n}$ inside $C$. The Weil pairing tells us that the dual isogeny $\widehat{\phi}: D \rightarrow C$ has kernel a labelled copy of $\mathbf{Z} / n \mathbf{Z}$ inside $D$. We estimate the Mordell-Weil rank by bounding the groups $D(K) / \phi C(K)$ and $C(K) / \widehat{\phi} D(K)$. This process is known as descent via $n$-isogeny. In many cases it enables us to compute the $n$-torsion of the Tate-Shafarevich groups $\amalg(C / K)$ and $\amalg(D / K)$.

Selmer wrote down the following example of a plane cubic which violates the Hasse principle.

$$
T=\left\{3 x_{0}^{3}+4 x_{1}^{3}+5 x_{2}^{3}=0\right\} \subset \mathbf{P}^{2} .
$$

As explained in [Ca3], $T$ corresponds to an element of order 3 in $\amalg(C / \mathbf{Q})$ where the Jacobian $C$ has equation $x_{0}^{3}+x_{1}^{3}+60 x_{2}^{3}=0$. There is an action of $\mu_{3}$ on $T$ given by $x_{i} \mapsto \zeta_{3}^{i} x_{i}$. The quotient is the elliptic curve $D$ with Weierstrass equation

$$
y^{2}=x^{3}+30^{2}
$$

A 2-descent shows $D(\mathbf{Q}) \cong \mathbf{Z} / 3 \mathbf{Z}$. Writing down explicit equations for the quotient map it is then easy to check $T(\mathbf{Q})=\emptyset$.

We now give an example of an element of order 5 in the Tate-Shafarevich group.

$$
T=\left\{\begin{array}{r}
x_{0}^{2}+x_{1} x_{4}-6 x_{2} x_{3}=0 \\
x_{1}^{2}+x_{0} x_{2}-15 x_{3} x_{4}=0 \\
2 x_{2}^{2}+x_{1} x_{3}-5 x_{0} x_{4}=0 \\
3 x_{3}^{2}+x_{2} x_{4}-x_{0} x_{1}=0 \\
5 x_{4}^{2}+x_{0} x_{3}-2 x_{1} x_{2}=0
\end{array}\right\} \subset \mathbf{P}^{4} .
$$

To show $T$ has points everywhere locally it suffices to consider the primes $p=$ $2,3,5,569$, at all other primes the reduction being a smooth curve of genus 1 . For $p=2,3,5$ the reduction is a rational nodal curve of degree 5 and again local solubility is clear. Finally $T$ meets the hyperplane $\left\{x_{4}=0\right\}$ in a point defined over $\mathbf{Q}(\sqrt[5]{12})$ and $p=569$ splits in this field. To show $T$ has no Q-rational points we quotient out by the action of $\mu_{5}$ on $T$ given by $x_{i} \mapsto \zeta_{5}^{i} x_{i}$. The quotient is an 
elliptic curve $D$ with Weierstrass equation

$$
y^{2}-29 x y-30 y=x^{3}-30 x^{2} .
$$

A 2-descent, courtesy of Cremona's program mwrank, shows $D(\mathbf{Q}) \cong \mathbf{Z} / 5 \mathbf{Z}$. It is then easy to check $T(\mathbf{Q})=\emptyset$.

The curve $T$ corresponds to an element of order 5 in $\amalg(C / \mathbf{Q})$ where the Jacobian $C$ is related to $D$ by the exact sequences (1). In our example $C$ and $D$ have conductor $N=17070$. We perform similar calculations for all pairs of elliptic curves $C$ and $D$ appearing in Cremona's tables [Cr3] of elliptic curves of conductor $N \leq 5300$. We find the following examples of curves $C$ with $\mathrm{II}(C / \mathbf{Q})[5] \cong(\mathbf{Z} / 5 \mathbf{Z})^{2}$

570L3, 570L4, 870I3, 870I4, 1050O2, 1342C3, 1938J2, 1950Y2, 2370M2, 2550EE2, 3270H2.

We are able to treat the case $n=7$ in the same explicit manner, and find the following examples of curves $C$ with $\amalg(C / \mathbf{Q})[7] \cong(\mathbf{Z} / 7 \mathbf{Z})^{2}$

$546 \mathrm{~F} 2,858 \mathrm{~K} 2,1230 \mathrm{~K} 2,5010 \mathrm{H} 2$.

It is pleasing to note that, with one exception, we have recovered Cremona's list of all curves of conductor $N \leq 1000$ and $\amalg$ of analytic order $n^{2}$. The exceptional curve 275B3, with $\amalg$ of analytic order 25 , is the 5-twist of one of our curves $C$. We are also able to exhibit examples of non-trivial $\amalg$ for elliptic curves with positive Mordell-Weil rank.

Let us make two remarks that may help the reader. Firstly, as the title of this article indicates, we often treat the cases $n=5$ and $n=7$ concurrently. It then becomes necessary, when giving formulae, to split into these two cases. At times we tire of reminding the reader that these are the cases $n=5$ and $n=7$. It is hoped that this will cause no confusion.

Secondly, we encounter many calculations that are straightforward to check with the aid of any computer algebra package, but which would be rather laborious to check by hand. The reader should either take these results on trust, or reach for his own computer. Whenever our computer calculations extend beyond these menial algebraic manipulations, we give explicit mention of the fact. For example in Sect. 1.4 we use Cremona's program mwrank which computes ranks of elliptic curves over $\mathbf{Q}$ via 2-descent.

\section{Descent calculations}

\subsection{Weierstrass equations and reduction types}

Let $K$ be a number field and $n \geq 4$ an integer. We consider pairs of $n$-isogenous elliptic curves $C$ and $D$ over $K$. We assume $\phi: C \rightarrow D$ has kernel a labelled copy of $\boldsymbol{\mu}_{n}$ and $\widehat{\phi}: D \rightarrow C$ has kernel a labelled copy of $\mathbf{Z} / n \mathbf{Z}$. The pairs of such curves $C$ and $D$ are parametrised by the modular curve $Y_{1}(n)$. For $n$ prime, $X_{1}(n)$ has $n-1$ cusps, of which half are defined over $\mathbf{Q}$ and half are defined over $\mathbf{Q}\left(\boldsymbol{\mu}_{n}\right) \cap \mathbf{R}$. 
Our interest is in the cases $n=5$ and $n=7$ when $X_{1}(n) \cong \mathbf{P}^{1}$. We shall shortly specify a co-ordinate $\lambda$ on $X_{1}(n)$ and write $C_{\lambda}$ and $D_{\lambda}$ for the pair of $n$-isogenous elliptic curves above $\lambda$.

Lemma 1.1. Let $E / K$ be an elliptic curve and $P \in E(K)$ a point of order at least 4 .

(i) E has a Weierstrass equation $y^{2}+u x y+v y=x^{3}+v x^{2}$ with $P=(0,0)$.

(ii) The pair $(E, P)$ uniquely determines $(u, v) \in \mathbf{A}^{2}(K)$.

(iii) The pair $(E, P)$ has no automorphisms.

Proof. [Si1, Exercise 8.13] or [Si2, Exercise 3.1].

We now compute $m P=\left(x_{m}(u, v), y_{m}(u, v)\right)$ for small integers $m$. Equating some of these expressions we quickly write down some affine curves whose smooth projective model is $X_{1}(n)$. In the cases $n=5$ and $n=7$ we find $D_{\lambda}$ has Weierstrass equation

$$
\begin{aligned}
& n=5 \quad y^{2}+(1-\lambda) x y-\lambda y=x^{3}-\lambda x^{2} \\
& n=7 \quad y^{2}+\left(1+\lambda-\lambda^{2}\right) x y+\left(\lambda^{2}-\lambda^{3}\right) y=x^{3}+\left(\lambda^{2}-\lambda^{3}\right) x^{2} .
\end{aligned}
$$

Computing the discriminant we find the cusps of $X_{1}(n)$.

$$
\begin{array}{ll}
n=5 & \begin{array}{l}
\text { Rational cusps at } \lambda=0, \infty \\
\text { Irrational cusps at the roots of } \lambda^{2}-11 \lambda-1=0
\end{array} \\
n=7 & \begin{array}{l}
\text { Rational cusps at } \lambda=0,1, \infty \\
\text { Irrational cusps at the roots of } \lambda^{3}-8 \lambda^{2}+5 \lambda+1=0
\end{array}
\end{array}
$$

The automorphisms of $Y_{1}(n)$ are precisely the automorphisms of $\mathbf{P}^{1}$ that map cusps to cusps. There are $(n-1) / 2$ automorphisms preserving the rational cusps. These correspond to relabelling our copy of $\boldsymbol{\mu}_{n} \hookrightarrow C$ respectively $\mathbf{Z} / n \mathbf{Z} \hookrightarrow D$. In addition there is an involution $\eta$ that swaps over the rational cusps with the irrational cusps. We find $\eta$ is defined over $\mathbf{Q}\left(\boldsymbol{\mu}_{n}\right) \cap \mathbf{R}$ and $\boldsymbol{\mu}_{n} \hookrightarrow C_{\lambda}$ is isomorphic to $\mathbf{Z} / n \mathbf{Z} \hookrightarrow D_{\eta(\lambda)}$ over $\mathbf{Q}\left(\boldsymbol{\mu}_{n}\right)$. Since there is no canonical choice of isomorphism $\boldsymbol{\mu}_{n} \cong \mathbf{Z} / n \mathbf{Z}$ there is no canonical choice of involution $\eta$. As far as possible we shall avoid making such a choice.

Remark 1.2. The proof of Lemma 1.1 involves writing down a Weierstrass equation and then making substitutions of the form

$$
x \mapsto \pi^{2} x+r, \quad y \mapsto \pi^{3} y+\pi^{2} s x+t .
$$

By this method we may easily check that for $n=5,\left(D_{\lambda}, 2 P\right) \cong\left(D_{-1 / \lambda}, P\right)$, and for $n=7,\left(D_{\lambda}, 2 P\right) \cong\left(D_{(\lambda-1) / \lambda}, P\right)$.

Vélu's formulae [V] tell us that $C_{\lambda}$ has Weierstrass equation

$$
\begin{aligned}
y^{2}+(1-\lambda) x y-\lambda y & =x^{3}-\lambda x^{2}-5 t x-b_{2} t-7 w \\
y^{2}+\left(1+\lambda-\lambda^{2}\right) x y+\left(\lambda^{2}-\lambda^{3}\right) y & =x^{3}+\left(\lambda^{2}-\lambda^{3}\right) x^{2}-5 t x-b_{2} t-7 w
\end{aligned}
$$


where

$$
\begin{aligned}
& n=5 \quad\left\{\begin{aligned}
b_{2} & =\lambda^{2}-6 \lambda+1 \\
t & =\lambda\left(\lambda^{2}+2 \lambda-1\right) \\
w & =\lambda^{2}\left(2 \lambda^{2}+\lambda+1\right)
\end{aligned}\right. \\
& n=7 \quad\left\{\begin{aligned}
b_{2} & =\lambda^{4}-6 \lambda^{3}+3 \lambda^{2}+2 \lambda+1 \\
t & =\lambda(\lambda-1)\left(\lambda^{2}-\lambda+1\right)\left(\lambda^{3}+2 \lambda^{2}-5 \lambda+1\right) \\
w & =\lambda^{2}(\lambda-1)^{2}\left(2 \lambda^{6}-2 \lambda^{5}+\lambda^{4}-8 \lambda^{3}+15 \lambda^{2}-9 \lambda+2\right) .
\end{aligned}\right.
\end{aligned}
$$

The Weierstrass equations (2) and (3) have discriminant

$$
\begin{array}{ll}
n=5 & \Delta\left(C_{\lambda}\right)=\lambda\left(\lambda^{2}-11 \lambda-1\right)^{5} \\
& \Delta\left(D_{\lambda}\right)=\lambda^{5}\left(\lambda^{2}-11 \lambda-1\right) \\
n=7 & \Delta\left(C_{\lambda}\right)=\lambda(\lambda-1)\left(\lambda^{3}-8 \lambda^{2}+5 \lambda+1\right)^{7} \\
& \Delta\left(D_{\lambda}\right)=\lambda^{7}(\lambda-1)^{7}\left(\lambda^{3}-8 \lambda^{2}+5 \lambda+1\right)
\end{array}
$$

Although our descent calculations shall make remarkably little explicit use of the reduction types for $C_{\lambda}$ and $D_{\lambda}$, it is good culture to describe them now. We recall that isogenous elliptic curves always have the same reduction type. Applying Tate's algorithm, as described in [Si2] for example, we find

Lemma 1.3. Let $\mathfrak{p}$ be a prime of $K$ with $\mathfrak{p} \nmid n$. For $\lambda \in K_{\mathfrak{p}}$ with $\operatorname{ord}_{\mathfrak{p}}(\lambda) \geq 0$ the Weierstrass equations (2) and (3) are minimal and the reduction types are as follows.

(i) If $\lambda$ reduces to a rational cusp, i.e. $\lambda \equiv 0(\bmod \mathfrak{p})$, respectively $\lambda(\lambda-1) \equiv 0$ $(\bmod \mathfrak{p})$, then $C_{\lambda}$ and $D_{\lambda}$ have split multiplicative reduction.

(ii) If $\lambda$ reduces to an irrational cusp, i.e. $\lambda^{2}-11 \lambda-1 \equiv 0(\bmod \mathfrak{p})$, respectively $\lambda^{3}-8 \lambda^{2}+5 \lambda+1 \equiv 0(\bmod \mathfrak{p})$, then $C_{\lambda}$ and $D_{\lambda}$ have multiplicative reduction and the reduction is split if and only if $\operatorname{Norm} \mathfrak{p} \equiv 1(\bmod n)$.

(iii) In the remaining cases $C_{\lambda}$ and $D_{\lambda}$ have good reduction.

The assumption $\operatorname{ord}_{\mathfrak{p}}(\lambda) \geq 0$ here is no loss since we are always free to replace $\lambda$ by $-1 / \lambda$, respectively $(\lambda-1) / \lambda$. It remains to describe the reduction types for $\mathfrak{p} \mid n$. We find that cases (i) and (iii) go through as before. However if $\lambda$ reduces to an irrational cusp it seems no longer possible to treat all number fields at once. If $K=\mathbf{Q}$ the Weierstrass equation (2) is still minimal and the reduction is additive. However, in this case the Weierstrass equation (3) need not be minimal, specifically if $\lambda \equiv 18(\bmod 25)$, respectively $\lambda \equiv 5(\bmod 7)$.

\subsection{Selmer groups and torsors}

Let $E$ be an elliptic curve over a number field $K$. Let $T / K$ be a smooth curve of genus 1 . We say that $T$ has the structure of torsor under $E$ if there is a simple transitive action $E \times T \rightarrow T$ defined over $K$. Since $T$ has $\bar{K}$-rational points, it is clear that $T$ is a twist of $E$. It is a general principle that the twists of an object $X$ are parametrised by $H^{1}(K, \operatorname{Aut}(X)):=H^{1}(\operatorname{Gal}(\bar{K} / K), \operatorname{Aut}(X))$. 
Writing $\tau_{P}$ for translation by $P \in E$ there is an exact sequence

$$
0 \rightarrow\left\{\text { translations } \tau_{P}\right\} \rightarrow \operatorname{Aut}(E) \rightarrow \operatorname{Aut}(E, 0) \rightarrow 0
$$

where $\operatorname{Aut}(E)$ and $\operatorname{Aut}(E, 0)$ are the automorphism groups of $E$ as a curve and as a group variety respectively. The translations are the automorphisms of $E$ as a torsor under itself. Thus the torsors under $E$ are parametrised by the Weil-Châtelet group $\mathrm{WC}(E / K):=H^{1}(K, E)$. Concretely, $T$ is a torsor under $E$ if and only if there exists $\psi: T \stackrel{\sim}{\longrightarrow} E$ an isomorphism over $\bar{K}$ such that the cocycle $\sigma \mapsto \sigma(\psi) \psi^{-1}$ takes values in the translation subgroup of $\operatorname{Aut}(E)$. We remark that in this situation the curve $T$ uniquely determines the curve $E$. In fact $E$ is the Jacobian of $T$.

We write $\xi_{T} \in \mathrm{WC}(E / K)$ for the cohomology class determined by the torsor $T / K$. It is clear $T(K) \neq \varnothing$ if and only if $\xi_{T}=0$. Thus the Tate-Shafarevich group

$$
\amalg(E / K):=\operatorname{ker}\left(\mathrm{WC}(E / K) \rightarrow \prod_{v} \mathrm{WC}\left(E / K_{v}\right)\right)
$$

measures the failure of the Hasse Principle for torsors $T$ under $E$.

We now take $\lambda \in K$, not a cusp of $X_{1}(n)$, and consider the elliptic curves $C_{\lambda}$ and $D_{\lambda}$ introduced in Sect. 1.1. There is an exact sequence of Galois modules

$$
0 \rightarrow \mu_{n} \rightarrow C_{\lambda} \rightarrow D_{\lambda} \rightarrow 0
$$

Taking Galois cohomology yields

$$
0 \rightarrow D_{\lambda}(K) / \phi C_{\lambda}(K) \stackrel{\delta}{\rightarrow} H^{1}\left(K, \mu_{n}\right) \rightarrow \mathrm{WC}\left(C_{\lambda} / K\right)[\phi] \rightarrow 0
$$

and by Hilbert's Theorem 90 we identify $H^{1}\left(K, \mu_{n}\right)=K^{\times} /\left(K^{\times}\right)^{n}$. We write $C_{\lambda, \theta}$ for the torsor under $C_{\lambda}$ described by $\theta \in K^{\times} /\left(K^{\times}\right)^{n}$. The Selmer group attached to the isogeny $\phi$ is defined by

$$
S^{(\phi)}\left(C_{\lambda} / K\right)=\left\{\theta \in K^{\times} /\left(K^{\times}\right)^{n} \mid C_{\lambda, \theta}\left(K_{\mathfrak{p}}\right) \neq \emptyset \text { for all primes } \mathfrak{p}\right\} .
$$

Notice that, since $n$ is odd, we are able to ignore the infinite places. We give equations for the curves $C_{\lambda, \theta}$ via two different methods, which we refer to as the push-out method and the projective space method.

Equations via Push-Out. Let $C_{\lambda}$ and $D_{\lambda}$ be as above and write 1 for the generator of $\mathbf{Z} / n \mathbf{Z} \hookrightarrow D_{\lambda}(K)$. We label our copy of $\boldsymbol{\mu}_{n} \hookrightarrow C_{\lambda}$ such that $e_{\phi}(\zeta, \mathbf{1})=\zeta$ for all $\zeta \in \mu_{n}$. Here $e_{\phi}$ is the Weil pairing as defined in [Si1, Exercise 3.15].

Lemma 1.4. Let $f \in K\left(D_{\lambda}\right)$ have divisor $(f)=n .1-n .0$ and be scaled, up to $n$th powers, such that $f_{\circ} \phi=g^{n}$ for some $g \in K\left(C_{\lambda}\right)$. Then the map $\delta$ in

$$
0 \rightarrow \mu_{n}(K) \rightarrow C_{\lambda}(K) \stackrel{\phi}{\rightarrow} D_{\lambda}(K) \stackrel{\delta}{\rightarrow} K^{\times} /\left(K^{\times}\right)^{n}
$$

is given by $\delta(P)=f(P) \bmod \left(K^{\times}\right)^{n}$, for $P \neq 0, \mathbf{1}$. 
Proof. Pick $P^{\prime} \in C_{\lambda}$ with $\phi\left(P^{\prime}\right)=P$. For $\sigma \in G_{K}:=\operatorname{Gal}(\bar{K} / K)$ we compute

$$
\begin{aligned}
\sigma\left(P^{\prime}\right)-P^{\prime} & =e_{\phi}\left(\sigma\left(P^{\prime}\right)-P^{\prime}, \mathbf{1}\right) \\
& =g\left(X+\sigma\left(P^{\prime}\right)-P^{\prime}\right) / g(X) \quad \text { for any } X \in C_{\lambda} \\
& =\sigma\left(g\left(P^{\prime}\right)\right) / g\left(P^{\prime}\right) .
\end{aligned}
$$

Thus $\delta(P)=g\left(P^{\prime}\right)^{n}=f_{\circ} \phi\left(P^{\prime}\right)=f(P) \bmod \left(K^{\times}\right)^{n}$.

Taking $D_{\lambda}$ with Weierstrass equation (2) and $\mathbf{1}=(0,0)$ we claim that the rational function $f \in K\left(D_{\lambda}\right)$ is given by

$$
\begin{array}{ll}
n=5 & f(x, y)=x y+y-x^{2} \\
n=7 & f(x, y)=(\lambda+1) x^{3}-x^{2} y+\lambda^{2} x^{2}-(2 \lambda+1) x y-\lambda^{2} y .
\end{array}
$$

Indeed on the affine piece of $D_{\lambda}$ described, $f$ only vanishes at $\mathbf{1}=(0,0)$, as is seen by computing some resultants. It follows that $f$ has the correct divisor. Since $\delta$ is a homomorphism it is easy to check that $f$ is correctly scaled. To this end we compute $f$ on the torsion $\mathbf{Z} / n \mathbf{Z} \hookrightarrow D_{\lambda}(K)$.

\begin{tabular}{c|ccc} 
& $x$ & $y$ & $f$ \\
\hline $\mathbf{2}$ & $\lambda$ & $\lambda^{2}$ & $\lambda^{3}$ \\
$\mathbf{3}$ & $\lambda$ & 0 & $-\lambda^{2}$ \\
$\mathbf{4}$ & 0 & $\lambda$ & $\lambda$
\end{tabular}

\begin{tabular}{c|ccc} 
& $x$ & $y$ & $f$ \\
\hline $\mathbf{2}$ & $\lambda^{2}(\lambda-1)$ & $\lambda^{3}(\lambda-1)^{2}$ & $-\lambda^{6}(\lambda-1)^{5}$ \\
$\mathbf{3}$ & $\lambda(\lambda-1)$ & $\lambda(\lambda-1)^{2}$ & $\lambda^{2}(\lambda-1)^{4}$ \\
$\mathbf{4}$ & $\lambda(\lambda-1)$ & $\lambda^{2}(\lambda-1)^{2}$ & $-\lambda^{5}(\lambda-1)^{3}$ \\
$\mathbf{5}$ & $\lambda^{2}(\lambda-1)$ & 0 & $\lambda^{8}(\lambda-1)^{2}$ \\
$\mathbf{6}$ & 0 & $\lambda^{2}(\lambda-1)$ & $-\lambda^{4}(\lambda-1)$
\end{tabular}

Thus $C_{\lambda, \theta}$ has affine model

$$
\left\{\begin{array}{c}
f(x, y)=\theta z^{n} \\
(x, y) \in D_{\lambda}
\end{array}\right\} \subset \mathbf{A}^{2} \times \mathbf{G}_{m} .
$$

Equations in Projective Space. We recall [Si1, Chapter III] that for D a divisor on the elliptic curve $E$

$$
D \sim 0 \Longleftrightarrow \operatorname{deg} D=0 \text { and } \operatorname{sum} D=0 .
$$

Thus if we embed $E \hookrightarrow \mathbf{P}^{n-1}$ by means of a complete linear system of degree $n$ then the translation map $\tau_{P}$ lifts to an automorphism of $\mathbf{P}^{n-1}$ if and only if $P \in E[n]$.

Now take $T \hookrightarrow \mathbf{P}^{n-1}$ a smooth curve of genus 1 and degree $n$. In this situation we always take it as read that $T$ is embedded via a complete linear system. It is equivalent to demand that $T$ is contained in no hyperplane. We claim that the action of $\operatorname{Jac}(T)$ on $T$ determines an inclusion of $G_{K}$-modules $\operatorname{Jac}(T)[n] \hookrightarrow \mathrm{PGL}_{n}$. Indeed, from a geometric point of view this situation is no different from that described in the last paragraph. 
Suppose given $T$ a torsor under $C_{\lambda}$ with $\xi_{T} \in \mathrm{WC}\left(C_{\lambda} / K\right)[\phi]$. We know $\mu_{n} \hookrightarrow C_{\lambda}$ acts on $T$ with quotient $U$ a torsor under $D_{\lambda}$. Then

$$
\phi: \mathrm{WC}\left(C_{\lambda} / K\right) \rightarrow \mathrm{WC}\left(D_{\lambda} / K\right) ; \xi_{T} \mapsto \xi_{U}=0 .
$$

Choosing a $K$-point on $U$ gives a divisor of degree $n$ on $T$ and so an embedding $T \hookrightarrow \mathbf{P}^{n-1}$. As explained above, $\boldsymbol{\mu}_{n} \hookrightarrow C_{\lambda}$ lifts to an action on $\mathbf{P}^{n-1}$. Geometrically there are exactly $n$ hyperplanes fixed under this action. They correspond to our original choice of $K$-point on $U$ and its translates under $\mathbf{Z} / n \mathbf{Z} \hookrightarrow D_{\lambda}$. In particular these hyperplanes are defined over $K$, so for a suitable choice of co-ordinates on $\mathbf{P}^{n-1}$ the action $\boldsymbol{\mu}_{n} \hookrightarrow \mathrm{PGL}_{n}$ is given by

$$
\zeta \mapsto \operatorname{Diag}\left(1: \zeta: \ldots: \zeta^{n-1}\right)
$$

Thus the torsors $C_{\lambda, \theta}$ all arise as smooth curves of genus 1 , degree $n$ in $\mathbf{P}^{n-1}$ invariant under (12). In Sect. 2.2 we shall give equations for all such curves. In the meantime, let us reverse the above argument to show that all the curves we obtain are of the form $C_{\lambda, \theta}$ for suitable $\lambda$ and $\theta$.

So let $T$ be a smooth curve of genus 1, degree $n$ in $\mathbf{P}^{n-1}$ invariant under the diagonal action of $\boldsymbol{\mu}_{n}$ given by (12). Since $n$ is prime to 6 we know $\boldsymbol{\mu}_{n} \hookrightarrow \operatorname{Jac}(T)$ and so $\operatorname{Jac}(T) \cong C_{\lambda}$ for some $\lambda \in K$. Now $T$ is a torsor under $C_{\lambda}$ and $U:=T / \mu_{n}$ is a torsor under $D_{\lambda}$. But the intersection of $T$ with one of the co-ordinate hyperplanes is an orbit under $\mu_{n}$ globally defined over $K$. Thus $U$ is trivial as a torsor under $D_{\lambda}$ and $\xi_{T} \in \mathrm{WC}\left(C_{\lambda} / K\right)[\phi]$. From the exact sequence (7) it follows $T \cong C_{\lambda, \theta}$ for some $\theta \in K^{\times} /\left(K^{\times}\right)^{n}$.

Remark 1.5. The action (12) of $\boldsymbol{\mu}_{n}$ on $\mathbf{P}^{n-1}$ is the unique one that lifts to GL ${ }_{n}$. By unique here we mean unique up to change of co-ordinates defined over $K$. In general the obstruction to lifting $\boldsymbol{\mu}_{n} \hookrightarrow \mathrm{PGL}_{n}$ to $\boldsymbol{\mu}_{n} \hookrightarrow \mathrm{GL}_{n}$ is given by $H^{1}(K, \mathbf{Z} / n \mathbf{Z})$.

Remark 1.6. The above arguments show that the torsors parametrised by $\mathrm{WC}(C / K)[\phi]$ may be embedded in $\mathbf{P}^{n-1}$. This is also known for the torsors parametrised by $\amalg(C / K)[n]$, and we suspect the local assumptions here are essential. We refer to $[\mathrm{CM}]$ where it is explained how this result is a consequence of the Hasse Principle for Brauer-Severi varieties.

\subsection{Statement of the descent theorem}

Let $n=5$ or 7 and let $\lambda \in \mathbf{Q}$ with $\lambda \neq 0$, respectively $\lambda \neq 0,1$. We consider the elliptic curves $C_{\lambda}$ and $D_{\lambda}$ over $\mathbf{Q}$ defined by (3) and (2). In each case $\mathbf{Z} / n \mathbf{Z} \hookrightarrow D_{\lambda}$ is generated by $(x, y)=(0,0)$ and the isogenous curve $C_{\lambda}$ is defined as the quotient by this subgroup. We write $\phi: C_{\lambda} \rightarrow D_{\lambda}$ for the $n$-isogeny with $C[\phi] \cong \mu_{n}$ and $D[\widehat{\phi}] \cong \mathbf{Z} / n \mathbf{Z}$. In this section we state our main results describing the Selmer groups $S^{(\phi)}\left(C_{\lambda} / \mathbf{Q}\right)$ and $S^{(\widehat{\phi})}\left(D_{\lambda} / \mathbf{Q}\right)$.

To help us carry the cases $n=5$ and $n=7$ together we write $\alpha(\lambda)$ and $\beta(\lambda)$ for the polynomials

$$
\alpha(\lambda)=\left\{\begin{array}{l}
\lambda \\
\lambda^{4}(\lambda-1)
\end{array} \quad \beta(\lambda)=\left\{\begin{array}{l}
\lambda^{2}-11 \lambda-1 \\
\lambda^{3}-8 \lambda^{2}+5 \lambda+1
\end{array}\right.\right.
$$


The zeros of these polynomials are cusps of $X_{1}(n)$. We define disjoint sets of rational primes.

$$
\begin{aligned}
& \mathcal{A}=\left\{p \text { prime } \mid \operatorname{ord}_{p}(\lambda)<0 \text { or } \alpha(\lambda) \equiv 0(\bmod p)\right\} \\
& \mathcal{B}=\left\{p \text { prime } \mid \begin{array}{c}
\beta(\lambda) \equiv 0(\bmod p) \text { and } p \equiv 1 \quad(\bmod n) \\
\text { or } p=n=5 \text { and } \lambda \equiv 18(\bmod 25) \\
\text { or } p=n=7 \text { and } \lambda \equiv 5(\bmod 7)
\end{array}\right\} .
\end{aligned}
$$

For $\mathcal{S}$ a set of rational primes, we write $[\mathcal{S}]$ for the subspace of $\mathbf{Q}^{\times} /\left(\mathbf{Q}^{\times}\right)^{n}$ generated by $\mathcal{S}$. For $\Xi$ a pairing of $\mathbf{Z} / n \mathbf{Z}$-vector spaces we write $\operatorname{ker}_{L}(\Xi)$ and $\operatorname{ker}_{R}(\Xi)$ for the left and right kernels.

Theorem 1. Let $n=5$ or 7 and let $\lambda \in \mathbf{Q}$ with $\lambda \neq 0$, respectively $\lambda \neq 0,1$. Let $\mathcal{A}$ and $\mathcal{B}$ be the disjoint sets of rational primes defined above. Then the Selmer group attached to the n-isogeny $\phi: C_{\lambda} \rightarrow D_{\lambda}$ is given by

$$
S^{(\phi)}\left(C_{\lambda} / \mathbf{Q}\right) \cong\left\{\theta \in[\mathcal{A}] \mid \theta \in\left(\mathbf{Q}_{p}^{\times}\right)^{n} \text { for all } p \in \mathcal{B}\right\} .
$$

The contribution to $S^{(\phi)}\left(C_{\lambda} / \mathbf{Q}\right)$ coming from $\mathbf{Z} / n \mathbf{Z} \hookrightarrow D_{\lambda}(\mathbf{Q})$ is generated by $\alpha(\lambda)$. Furthermore there is a pairing $\Xi:[\mathcal{A}] \times[\mathcal{B}] \rightarrow \mathbf{Z} / n \mathbf{Z}$ such that $S^{(\phi)}\left(C_{\lambda} / \mathbf{Q}\right) \cong$ $\operatorname{ker}_{L}(\Xi)$ and $S^{(\widehat{\phi})}\left(D_{\lambda} / \mathbf{Q}\right) \cong \operatorname{ker}_{R}(\Xi)$.

Remark 1.7. The pairing $\Xi$ is straightforward to compute. For each prime $q \in \mathcal{B}$ we choose a non-trivial character $\chi_{q}:(\mathbf{Z} / q \mathbf{Z})^{\times} \rightarrow \mathbf{Z} / n \mathbf{Z}$, respectively $\chi_{n}:$

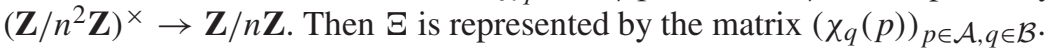

Lemma 1.8. For $p$ a rational prime, the congruence $\beta(\lambda) \equiv 0(\bmod p)$ is soluble if and only if $p=n$ or $p \equiv \pm 1(\bmod n)$.

Proof. Consider how $p$ factors in $\mathbf{Q}\left(\boldsymbol{\mu}_{n}\right) \cap \mathbf{R}$.

Remark 1.9. In view of the identities $\lambda^{2}-11 \lambda-1=(\lambda+7)(\lambda-18)+125$ and $\lambda^{3}-8 \lambda^{2}+5 \lambda+1=(\lambda+2)(\lambda-5)^{2}-49$, the condition for $n$ to belong to $\mathcal{B}$ is $\beta(\lambda) \equiv 0(\bmod 125)$, respectively $\beta(\lambda) \equiv 0(\bmod 49)$. As seen in Sect. 1.1 this is also the condition for the Weierstrass equation (3) to no longer be minimal.

We sketch the proof of Theorem 1. The definition (8) reads

$$
S^{(\phi)}\left(C_{\lambda} / \mathbf{Q}\right)=\left\{\theta \in \mathbf{Q}^{\times} /\left(\mathbf{Q}^{\times}\right)^{n} \mid C_{\lambda, \theta}\left(\mathbf{Q}_{p}\right) \neq \varnothing \text { for all primes } p\right\} .
$$

In Sect. 2.2 we give equations for the $C_{\lambda, \theta}$ as curves in $\mathbf{P}^{n-1}$. We then describe the geometry of the reductions $\bmod p$ and so derive some simple criteria for the existence of local points. We find that $S^{(\phi)}\left(C_{\lambda} / \mathbf{Q}\right)$ is the subspace of $[\mathcal{A}]$ consisting of $n$th powers modulo the primes in $\mathcal{B}$. To treat the dual isogeny $\widehat{\phi}$, the rough idea is that the involution $\eta$ swaps over the sets of primes $\mathcal{A}$ and $\mathcal{B}$. Then $S^{(\widehat{\phi})}\left(D_{\lambda} / \mathbf{Q}\right)$ is the subspace of $[\mathcal{B}]$ consisting of $n$th powers modulo the primes in $\mathcal{A}$, and the existence of the pairing $\Xi$ is nothing more than a statement of the $n$th power reciprocity law. To make these ideas precise it is natural to work over $K=\mathbf{Q}\left(\boldsymbol{\mu}_{n}\right)$. Since $[K: \mathbf{Q}]=n-1$ is prime to $n$, there is no difficulty in descending back to $\mathbf{Q}$. 
In this article we give a proof of Theorem 1 working entirely over $\mathbf{Q}$. In $[\mathrm{F}]$ we generalise Theorem 1 to $K=\mathbf{Q}\left(\boldsymbol{\mu}_{n}\right)$ and so give an alternative proof of the result over $\mathbf{Q}$ which is much closer to the above sketch.

We should mention that in the case $p=n$ the projective space method fails to give complete criteria for the existence of local points. Our solution is to revert to the push-out method and perform some further Hensel lemma calculations. It is these calculations which make it difficult to work over a general number field.

\subsection{Initial examples}

Let $n=5$ or 7 and let $\lambda \in \mathbf{Q}$ with $\lambda \neq 0$, respectively $\lambda \neq 0,1$. Theorem 1 gives us information about both the Mordell-Weil groups $C_{\lambda}(\mathbf{Q})$ and $D_{\lambda}(\mathbf{Q})$ and the Tate-Shafarevich groups $\amalg\left(C_{\lambda} / \mathbf{Q}\right)$ and $\amalg\left(D_{\lambda} / \mathbf{Q}\right)$. We now make this explicit. Following Mazur's classification of torsion groups for elliptic curves over $\mathbf{Q}$ we find

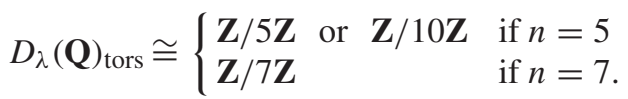

Let $r=\operatorname{rank} C_{\lambda}(\mathbf{Q})=\operatorname{rank} D_{\lambda}(\mathbf{Q})$ and $\iota=\operatorname{dim}_{n} C_{\lambda}(\mathbf{Q})[n]$. Then $\iota=0$ unless $\alpha(\lambda) \in\left(\mathbf{Q}^{\times}\right)^{n}$ and this can only happen in the case $n=5$. Now Theorem 1 together with the exact sequences

$$
\begin{aligned}
0 & \rightarrow D_{\lambda}(\mathbf{Q}) / \phi C_{\lambda}(\mathbf{Q}) \rightarrow S^{(\phi)}\left(C_{\lambda} / \mathbf{Q}\right) \rightarrow \amalg\left(C_{\lambda} / \mathbf{Q}\right)[\phi] \rightarrow 0 \\
0 & \rightarrow C_{\lambda}(\mathbf{Q}) / \widehat{\phi} D_{\lambda}(\mathbf{Q}) \rightarrow S^{(\widehat{\phi})}\left(D_{\lambda} / \mathbf{Q}\right) \rightarrow \amalg\left(D_{\lambda} / \mathbf{Q}\right)[\widehat{\phi}] \rightarrow 0
\end{aligned}
$$

tells us $r=r_{1}+r_{2}$ with $r_{1}, r_{2} \geq 0$ and

$$
\begin{aligned}
& |\mathcal{A}|-\operatorname{rank}(\Xi)=r_{1}+1-\iota+\operatorname{dim}_{n} \amalg\left(C_{\lambda} / \mathbf{Q}\right)[\phi] \\
& |\mathcal{B}|-\operatorname{rank}(\Xi)=r_{2}+\iota+\operatorname{dim}_{n} \amalg\left(D_{\lambda} / \mathbf{Q}\right)[\widehat{\phi}] .
\end{aligned}
$$

Thus our upper bound for the Mordell-Weil rank is

$$
|\mathcal{A}|+|\mathcal{B}|-1-2 \operatorname{rank}(\Xi) .
$$

In the following examples we make use of the trivial exact sequences

$$
\begin{aligned}
0 & \rightarrow \amalg\left(C_{\lambda} / \mathbf{Q}\right)[\phi] \rightarrow \amalg\left(C_{\lambda} / \mathbf{Q}\right)[n] \stackrel{\phi}{\rightarrow} \amalg\left(D_{\lambda} / \mathbf{Q}\right)[\widehat{\phi}] \\
0 & \rightarrow \amalg\left(D_{\lambda} / \mathbf{Q}\right)[\widehat{\phi}] \rightarrow \amalg\left(D_{\lambda} / \mathbf{Q}\right)[n] \stackrel{\widehat{\phi}}{\rightarrow} \amalg\left(C_{\lambda} / \mathbf{Q}\right)[\phi] .
\end{aligned}
$$

Example 1.10. Let $n=5$ and $\lambda=38$. Then $\mathcal{A}=\{2,19\}$ and $\mathcal{B}=\{41\}$. Since $2^{8} \not \equiv 1 \quad(\bmod 41)$ it is clear that $\Xi$ has rank 1 . By Theorem 1 we deduce $C_{38}(\mathbf{Q})=0, D_{38}(\mathbf{Q}) \cong \mathbf{Z} / 5 \mathbf{Z}$ and $\amalg\left(C_{38} / \mathbf{Q}\right)(5)=\amalg\left(D_{38} / \mathbf{Q}\right)(5)=0$.

Example 1.11. Let $n=7$ and $\lambda=8$. Then $\mathcal{A}=\{2,7\}$ and $\mathcal{B}=\emptyset$. A computer search yields the point of infinite order $(x, y)=(30,198)$ on $D_{8}$. By Theorem 1 we deduce $C_{8}(\mathbf{Q}) \cong \mathbf{Z}, D_{8}(\mathbf{Q}) \cong \mathbf{Z} / 7 \mathbf{Z} \oplus \mathbf{Z}$ and $\amalg\left(C_{8} / \mathbf{Q}\right)(7)=\amalg\left(D_{8} / \mathbf{Q}\right)(7)=0$. 
We may use Theorem 1 together with Cremona's program mwrank, which performs a 2-descent, to exhibit some elements of the Tate-Shafarevich group. As explained in the introduction these elements are explicit in the sense that we are able to give equations in $\mathbf{P}^{n-1}$ for the corresponding curves of genus 1 that violate the Hasse principle.

Example 1.12. Let $n=5$ and $\lambda=-60,-42,-30,30,60$ or 90 . In all these examples we have $|\mathcal{A}|=3$ and $\mathcal{B}=\emptyset$. According to mwrank, rank $D_{\lambda}(\mathbf{Q})=0$. By Theorem 1 we deduce $\amalg\left(C_{\lambda} / \mathbf{Q}\right)[5] \cong(\mathbf{Z} / 5 \mathbf{Z})^{2}$.

Example 1.13. Let $n=7$ and $\lambda=-6,-5,6,7,10$ or 11 . In all these examples $|\mathcal{A}|=3$ and $\mathcal{B}=\emptyset$. According to mwrank, $\operatorname{rank} D_{\lambda}(\mathbf{Q})=0$. By Theorem 1 we deduce $\amalg\left(C_{\lambda} / \mathbf{Q}\right)[7] \cong(\mathbf{Z} / 7 \mathbf{Z})^{2}$.

As promised in the introduction, we give some examples of non-trivial $\amalg$ for elliptic curves with positive Mordell-Weil rank.

Example 1.14. Let $n=5$ and $\lambda= \pm 30 / 7, \pm 35 / 6$ or $14 / 15$. In all these examples $\mathcal{A}=\{2,3,5,7\}$ and $\mathcal{B}=\emptyset$. According to mwrank, $\operatorname{rank} D_{\lambda}(\mathbf{Q})=1$. By Theorem 1 we deduce $\amalg\left(C_{\lambda} / \mathbf{Q}\right)[5] \cong(\mathbf{Z} / 5 \mathbf{Z})^{2}$.

\section{Geometric results}

\subsection{The geometry of elliptic normal curves}

Let $K$ be an algebraically closed field and $n \geq 3$ an integer. We assume char $(K) \nmid n$ and fix $\zeta=\zeta_{n}$ a primitive $n$th root of unity. We make further restrictions on $n$ in due course.

Definition 2.1. (i) An elliptic normal curve of degree $n$ is an elliptic curve $E \hookrightarrow \mathbf{P}^{n-1}$ of degree $n$ contained in no hyperplane.

(ii) A Néron polygon of degree $n$ is a collection of $n$ lines $\ell_{0}, \ldots, \ell_{n-1}$ in $\mathbf{P}^{n-1}$, contained in no hyperplane and arranged such that $\ell_{i}$ meets $\ell_{j}$ if and only if $i-j \equiv \pm 1(\bmod n)$.

Lemma 2.2. Let $E \hookrightarrow \mathbf{P}^{n-1}$ be either (i) an elliptic normal curve or (ii) a Néron polygon. Then $E$ lies on $n(n-3) / 2$ quadrics and for $n \geq 4$ these quadrics suffice to define $E$.

Proof. (i) See [H, Chapter IV].

(ii) We move the $n$ points of intersection to $(1: 0: 0: \ldots),(0: 1: 0: \ldots), \ldots$ and the result is clear.

The modular curve $Y(n)=X(n) \backslash$ cusps $\}$ parametrises the triples $(E, P, Q)$ where $E$ is an elliptic curve and $P, Q \in E[n]$ satisfy $e_{n}(P, Q)=\zeta_{n}$. For future reference we note there is an action of $\boldsymbol{\mu}_{n}$ on $X(n)$ given by

$$
\zeta_{n}:(E, P, Q) \mapsto(E, P, Q+P)
$$

with quotient $X_{1}(n)$. 
Proposition 2.3. Let $(E, P, Q)$ be as above. If we embed $E \hookrightarrow \mathbf{P}^{n-1}$ by means of a complete linear system, then we may choose co-ordinates on $\mathbf{P}^{n-1}$ such that the translation maps $\tau_{P}$ and $\tau_{Q}$ are given by

$$
M_{P}:=\left(\begin{array}{ccccc}
1 & 0 & 0 & \cdots & 0 \\
0 & \zeta & 0 & \cdots & 0 \\
0 & 0 & \zeta^{2} & \cdots & 0 \\
\vdots & \vdots & \vdots & & \vdots \\
0 & 0 & 0 & \cdots & \zeta^{n-1}
\end{array}\right) \quad M_{Q}:=\left(\begin{array}{ccccc}
0 & 0 & \cdots & 0 & 1 \\
1 & 0 & \cdots & 0 & 0 \\
0 & 1 & \cdots & 0 & 0 \\
\vdots & \vdots & & \vdots & \vdots \\
0 & 0 & \cdots & 1 & 0
\end{array}\right) .
$$

Proof. As explained in Sect. 1.2, $\tau_{P}$ and $\tau_{Q}$ lift to elements of $\operatorname{Aut}\left(\mathbf{P}^{n-1}\right)=\mathrm{PGL}_{n}$. Since no hyperplane may contain more than $n$ points of $E$ we deduce that $M_{P}$ has distinct eigenvalues and that $M_{Q}$ cyclically permutes the hyperplanes fixed by $M_{P}$. Thus we may choose co-ordinates on $\mathbf{P}^{n-1}$ such that $M_{P}$ and $M_{Q}$ are as given, at least if we replace $\zeta$ by $\zeta^{r}$ for some $r$ prime to $n$. We observe that $M_{P}$ and $M_{Q}$ commute as elements of $\mathrm{PGL}_{n}$, but do not commute when lifted to $\mathrm{GL}_{n}$. In fact their commutator in $\mathrm{GL}_{n}$ gives one possible definition of the Weil pairing. It follows that $r=1$ as required.

Proposition 2.4. Let $n \geq 3$ odd and let $(E, P, Q)$ be as above. If we embed $E \hookrightarrow \mathbf{P}^{n-1}$ by means of a complete linear system $|D|$ with $[-1]^{*} D \sim D$ then there is a unique choice of co-ordinates on $\mathbf{P}^{n-1}$ such that $\tau_{P}, \tau_{Q}$ and $[-1]$ are given by $M_{P}, M_{Q}$ and

$$
[-1]:=\left(\begin{array}{ccccc}
1 & 0 & \cdots & 0 & 0 \\
0 & 0 & \cdots & 0 & 1 \\
0 & 0 & \cdots & 1 & 0 \\
\vdots & \vdots & & \vdots & \vdots \\
0 & 1 & \cdots & 0 & 0
\end{array}\right)
$$

Proof. We first choose co-ordinates as described in Proposition 2.3. Since the subgroup generated by $M_{P}$ and $M_{Q}$ is its own centraliser inside $\mathrm{PGL}_{n}$ our coordinates are uniquely determined up to the action of this subgroup. We require

$$
[-1] M_{P}[-1]=M_{P}^{-1} \quad[-1] M_{Q}[-1]=M_{Q}^{-1} \quad \text { and } \quad[-1]^{2}=I .
$$

These commutator relations determine [-1] up to multiplication by elements of $\left\langle M_{P}, M_{Q}\right\rangle$. Since $n$ is odd, the $n^{2}$ possible matrices representing the map [-1] form a single orbit under the action of $\left\langle M_{P}, M_{Q}\right\rangle$ via conjugation. Taking [-1] as given now fixes our choice of co-ordinates.

We restrict to $n \geq 5$ odd, so that $E$ is defined by quadrics. For $(E, P, Q)$ a triple as above, we embed $E \hookrightarrow \mathbf{P}^{n-1}$ via the linear system $|n .0|$ and choose co-ordinates as in Proposition 2.4. Then $(E, P, Q)$ is uniquely determined by the co-ordinates of the point $0 \in \mathbf{P}^{n-1}$. Indeed, given the co-ordinates of this point, the action of $M_{P}$ and $M_{Q}$ allows us to write down $n^{2}$ points on $E$. But $E$ is defined by quadrics. So by Bézout's theorem these $n^{2}$ points suffice to determine $E$. Thus the above 
procedure gives an embedding $X(n) \hookrightarrow \mathbf{P}^{n-1}$. We shall describe the image of this map in the cases $n=5$ and 7 .

We write $x_{0}, x_{1}, \ldots, x_{n-1}$ for our co-ordinates on $\mathbf{P}^{n-1}$ and agree that all subscripts are to be read $\bmod n$. Our assumption $n$ is odd tells us

$$
n .0 \sim 0+P+2 P+\ldots+(n-1) P .
$$

Therefore 0 belongs to exactly one of the hyperplanes fixed by $M_{P}$. But 0 is fixed by $[-1]$ so we have either

$$
\text { or } \quad \begin{aligned}
& 0=\left(0: a_{1}: a_{2}: \ldots: a_{2}: a_{1}\right) \\
& \text { or } \quad\left(0: a_{1}: a_{2}: \ldots:-a_{2}:-a_{1}\right)
\end{aligned}
$$

for some $a_{1}, a_{2}, \ldots, a_{(n-1) / 2}$ non-zero. We consider the vector spaces

$$
V:=H^{0}\left(\mathbf{P}^{n-1}, \mathcal{I}_{E}(2)\right) \subset W:=H^{0}\left(\mathbf{P}^{n-1}, \mathcal{O}(2)\right) .
$$

The action of $M_{P}$ allows us to write these as direct sums $V=\oplus_{i} V_{i}$ and $W=\oplus_{i} W_{i}$ with $V_{i} \subset W_{i}=\left\langle x_{i}^{2}, x_{i-1} x_{i+1}, \ldots\right\rangle$. Since $n$ is odd we deduce via the action of $M_{Q}$ that $\operatorname{dim} V_{i}=(n-3) / 2$ and $\operatorname{dim} W_{i}=(n+1) / 2$. The point 0 and its translates under $M_{Q}$ impose some linear conditions on the coefficients of the quadrics in $V_{0}$. This leads us to rule out the case (+) and to make the following definition.

Definition 2.5. For $n \geq 5$ odd, let $A(n) \subset \mathbf{P}^{n-1}$ be the subscheme defined by $a_{0}=0, a_{n-i}=-a_{i}$ and

$$
\operatorname{rank}\left(a_{i-j} a_{i+j}\right)_{i, j=0}^{n-1} \leq 2 .
$$

More precisely $A(n)$ is defined by the leading $4 \times 4$ Pfaffians.

The above construction shows $X(n) \subset A(n)$. In fact, for $p$ a prime, it is a theorem of Vélu that $X(p)=A(p)$. In other words, the quartics (16) suffice to define $X(p)$. Since our interest is in the cases $n=5$ and 7 , there is no need to appeal to this general theorem.

Equations for $X(5)$. Let $0=(0: a: b:-b:-a)$. A(5) is defined by

$$
\operatorname{rank}\left(\begin{array}{ccc}
0 & -a^{2} & -b^{2} \\
a^{2} & 0 & a b \\
b^{2} & -a b & 0
\end{array}\right) \leq 2
$$

Thus $X(5)=A(5)$ is a copy of $\mathbf{P}^{1}$ and $V=\oplus_{i} V_{i}$ is spanned by

$$
a b x_{0}^{2}+b^{2} x_{1} x_{4}-a^{2} x_{2} x_{3}=0
$$

and cyclic permutes. There are cusps of $X(5)$ at $(a: b)=(1: 0),(0: 1)$. We call these the rational cusps. 
Equations for $X(7)$. Let $0=(0: a: b:-c: c:-b:-a)$. A(7) is defined by

$$
\operatorname{rank}\left(\begin{array}{cccc}
0 & -a^{2} & -b^{2} & -c^{2} \\
a^{2} & 0 & a c & -b c \\
b^{2} & -a c & 0 & a b \\
c^{2} & b c & -a b & 0
\end{array}\right) \leq 2 \quad \Longleftrightarrow \quad a^{3} b+b^{3} c+c^{3} a=0 .
$$

Thus $X(7)=A(7)$ is the Klein quartic [Kl] and $V=\oplus_{i} V_{i}$ is spanned by

$$
\begin{aligned}
a b x_{1} x_{6}+b c x_{2} x_{5}+c a x_{3} x_{4} & =0 \\
a b x_{0}^{2}+c^{2} x_{2} x_{5}-b^{2} x_{3} x_{4} & =0 \\
b c x_{0}^{2}-c^{2} x_{1} x_{6}+a^{2} x_{3} x_{4} & =0 \\
c a x_{0}^{2}+b^{2} x_{1} x_{6}-a^{2} x_{2} x_{5} & =0
\end{aligned}
$$

and cyclic permutes. There are cusps of $X(7)$ at $(a: b: c)=(1: 0: 0),(0: 1: 0)$, $(0: 0: 1)$. We call these the rational cusps.

Let $n=5$ or 7 . The equations (17) and (18) each define a family of curves $\mathcal{X}(n) \rightarrow X(n)$, whose fibres above $Y(n)$ are elliptic normal curves. Furthermore, there is an action of $\operatorname{PSL}_{2}(\mathbf{Z} / n \mathbf{Z})$ on $X(n)$ given by relabelling torsion, and this extends to an action of $\mathrm{SL}_{2}(\mathbf{Z} / n \mathbf{Z})$ on $\mathcal{X}(n)$. Directly from the equations we see that the fibres of $\mathcal{X}(n) \rightarrow X(n)$ above the rational cusps are Néron polygons. But the cusps form a single orbit under the action of $\mathrm{PSL}_{2}(\mathbf{Z} / n \mathbf{Z})$, so the same is true at all the cusps.

One way to find the irrational cusps is by explicitly writing down the action of $\mathrm{PSL}_{2}(\mathbf{Z} / n \mathbf{Z})$ on $X(n)$. We give an alternative method. We begin by computing the action (14) of $\mu_{n}$ on $X(n)$,

$$
n=5 \quad(a: b) \mapsto(\zeta a: b), \quad n=7 \quad(a: b: c) \mapsto\left(\zeta^{3} a: \zeta b: c\right) .
$$

Up to this action, the irrational cusps $\left(0: a_{1}: a_{2}: \ldots\right)$ satisfy

$$
\operatorname{rank}\left(a_{i-j}\right)_{i, j=0}^{n-1} \leq 2 .
$$

Indeed (20) may be viewed both as the condition for $\left(0: a_{1}: a_{2}: \ldots\right)$ and its translates under $M_{Q}$ to be collinear, and as the condition for $M_{Q}$ to fix a point on the fibre of $\mathcal{X}(n) \rightarrow X(n)$. Either of these conditions is sufficient to establish degeneration from an elliptic normal curve to a Néron polygon. Essentially by solving a second order recurrence relation, we find that the solutions to (20) with $a_{0}=0$ and $a_{n-i}=-a_{i}$ are

$$
\left(0: a_{1}: a_{2}: \ldots\right)=\left(0: \zeta-\zeta^{-1}: \zeta^{2}-\zeta^{-2}: \ldots\right)
$$

for $\zeta$ a primitive $n$th root of unity. Finally (19) and (21) allow us to list the irrational cusps.

Case $n=5$. The cusps are at $a / b=0, \infty, \zeta^{i} \phi, \zeta^{i} \bar{\phi}$ where $\phi$ is the golden ratio and $\bar{\phi}$ is its conjugate. These points may be viewed, under stereographic projection, as the vertices of an icosahedron. 
Case $n=7$. The irrational cusps are at

$$
(a: b: c)=\left(\zeta^{3 i}\left(\zeta-\zeta^{6}\right): \zeta^{i}\left(\zeta^{2}-\zeta^{5}\right): \zeta^{4}-\zeta^{3}\right)
$$

and its translates under $(a: b: c) \mapsto(c: a: b)$. Together with the rational cusps, these are the points of inflection of the Klein quartic [K1].

\subsection{Equations for torsors in $\mathbf{P}^{n-1}$}

Let $n=5$ or 7 . As explained in Sect. 1.2 we aim to give explicit equations for the torsors $C_{\lambda, \theta}$ as curves in $\mathbf{P}^{n-1}$. We work over a perfect field $K$ and until further notice assume $\operatorname{char}(K) \neq n$.

Let $T \hookrightarrow \mathbf{P}^{n-1}$ be a smooth curve of genus 1 and degree $n$ which is invariant under the action $\boldsymbol{\mu}_{n} \hookrightarrow \operatorname{Aut}\left(\mathbf{P}^{n-1}\right)$ given by

$$
\zeta \mapsto \operatorname{Diag}\left(1: \zeta: \ldots: \zeta^{n-1}\right)
$$

We write $\left(x_{0}: \ldots: x_{n-1}\right)$ for our co-ordinates on $\mathbf{P}^{n-1}$ and agree that all subscripts are to be read $\bmod n$. The hyperplanes fixed by (22) are the co-ordinate hyperplanes $H_{i}:=\left\{x_{i}=0\right\}$. As explained in Sect. 1.2 the action of $\operatorname{Jac}(T)[n]$ on $T$ lifts to $\mathbf{P}^{n-1}$. Since $n$ is prime to 6 we know $\mu_{n} \hookrightarrow \operatorname{Jac}(T)$ and so this action is generated by

$$
M_{1}=\left(\begin{array}{ccccc}
1 & 0 & 0 & \cdots & 0 \\
0 & \zeta & 0 & \cdots & 0 \\
0 & 0 & \zeta^{2} & \cdots & 0 \\
\vdots & \vdots & \vdots & & \vdots \\
0 & 0 & 0 & \cdots & \zeta^{n-1}
\end{array}\right) \quad M_{2}=\left(\begin{array}{ccccc}
0 & 0 & \cdots & 0 & * \\
* & 0 & \cdots & 0 & 0 \\
0 & * & \cdots & 0 & 0 \\
\vdots & \vdots & & \vdots & \vdots \\
0 & 0 & \cdots & * & 0
\end{array}\right)
$$

where $*$ denotes a non-zero element of $\bar{K}$. By Lemma 2.2 the curve $T$ is defined by 5 quadrics, respectively 14 quadrics. The action of $M_{1}$ divides these quadrics up into $n$-eigenspaces, and the action of $M_{2}$ tells us that these eigenspaces all have the same dimension. Concretely

$$
H^{0}\left(\mathbf{P}^{n-1}, \mathcal{I}_{T}(2)\right)=\oplus_{i} V_{i} \quad \text { with } \quad V_{i} \subset\left\langle x_{i}^{2}, x_{i-1} x_{i+1}, \ldots\right\rangle
$$

Lemma 2.6. Let $T \hookrightarrow \mathbf{P}^{n-1}$ satisfy the above hypotheses.

(i) The curve $T$ meets the hyperplanes $H_{i}$ in a total of $n^{2}$ points.

(ii) Every non-zero quadric containing $T$ has at least three non-zero terms.

Proof. (i) Let $P \in T \cap H_{0}$. Then the translates of $P$ under $M_{1}$ and $M_{2}$ give the required $n^{2}$ distinct points.

(ii) Suppose $V_{0}$ contains a quadric with exactly two non-zero terms. Then the $n^{2}$ points in (i) fail to be distinct. 
Definition 2.7. Let $\lambda, \tau_{0}, \ldots, \tau_{n-1} \in K$ satisfy $\alpha(\lambda)=\prod \tau_{i} \neq 0$. We define $T\left[\lambda ; \tau_{0}, \ldots, \tau_{n-1}\right]$ to be the subscheme of $\mathbf{P}^{n-1}$ with equations

$$
\begin{aligned}
& n=5 \quad \tau_{0} x_{0}^{2}+x_{1} x_{4}-\tau_{2} \tau_{3} x_{2} x_{3}=0 \quad \& \text { cyclic permutes } \\
& n=7\left\{\begin{array}{r}
\tau_{0} x_{0}^{2}+x_{1} x_{6}-\left(1 / \lambda^{2}\right) \tau_{2} \tau_{3} \tau_{4} \tau_{5} x_{2} x_{5}=0 \\
\tau_{0} x_{0}^{2}+\lambda x_{1} x_{6}-\left(1 / \lambda^{3}\right) \tau_{2} \tau_{3}^{2} \tau_{4}^{2} \tau_{5} x_{3} x_{4}=0
\end{array} \quad \&\right. \text { cyclic permutes. }
\end{aligned}
$$

Proposition 2.8. Every curve $T \hookrightarrow \mathbf{P}^{n-1}$ satisfying the above hypotheses is equal to $T\left[\lambda ; \tau_{0}, \ldots, \tau_{n-1}\right]$ for some $\lambda, \tau_{0}, \ldots, \tau_{n-1} \in K$.

Proof. (i) Case $n=5$. Lemma 2.6(ii) tells us that $T$ has equations

$$
\tau_{0} x_{0}^{2}+x_{1} x_{4}+a_{0} x_{2} x_{3}=0 \quad \text { \& cyclic permutes }
$$

for some non-zero constants $\tau_{i}, a_{i} \in K$. By Lemma 2.6(i) we may choose (0: $z_{1}$ : $\left.z_{2}: z_{3}: z_{4}\right) \in T \cap H_{0}$ with all $z_{i} \neq 0$. Then

$$
\left.\begin{array}{r}
z_{1} z_{4}+a_{0} z_{2} z_{3}=0 \\
\tau_{2} z_{2}^{2}+z_{1} z_{3}=0 \\
\tau_{3} z_{3}^{2}+z_{2} z_{4}=0
\end{array}\right\} \Longrightarrow a_{0}=-\tau_{2} \tau_{3} .
$$

By symmetry this completes the proof in the case $n=5$.

(ii) Case $n=7$. Lemma 2.6(ii) tells us that $T$ has equations

$$
\begin{aligned}
\tau_{0} x_{0}^{2}+x_{1} x_{6}+a_{0} x_{2} x_{5} & =0 \\
\tau_{0} x_{0}^{2}+\lambda_{0} x_{1} x_{6}+b_{0} x_{3} x_{4} & =0 \quad \text { \& cyclic permutes }
\end{aligned}
$$

for some non-zero constants $\tau_{i}, \lambda_{i}, a_{i}, b_{i} \in K$. The action of $M_{2}$ tells us $\lambda_{0}=$ $\lambda_{1}=\ldots=\lambda_{n-1}$ and we write $\lambda$ for the common value. Similarly, the action of $M_{2}$ suggests we write $a_{0}=a \tau_{2} \tau_{3} \tau_{4} \tau_{5}$ and $b_{0}=b \tau_{2} \tau_{3}^{2} \tau_{4}^{2} \tau_{5}$ since these relations will still hold when we cyclically permute the subscripts. It remains to see how the quantities $a, b, \lambda$ and $\prod \tau_{i}$ are related. By Lemma 2.6(i) we may choose $\left(0: z_{1}\right.$ : $\left.\ldots: z_{6}\right) \in T \cap H_{0}$ with all $z_{i} \neq 0$. We find

$$
\tau_{2} z_{2}^{2}+z_{1} z_{3}=\tau_{3} z_{3}^{2}+\lambda z_{2} z_{4}=\tau_{4} z_{4}^{2}+\lambda z_{3} z_{5}=\tau_{5} z_{5}^{2}+z_{4} z_{6}=0 .
$$

If $\prod z_{i}^{\mu_{i}}$ and $\prod z_{i}^{\nu_{i}}$ are two monomials with $\sum \mu_{i}=\sum v_{i}$ and $\sum i \mu_{i}=\sum i v_{i}$ then (23) allows us to express their ratio in terms of $\lambda$ and the $\tau_{i}$. In particular

$$
\begin{aligned}
& z_{1} z_{6}+a \tau_{2} \tau_{3} \tau_{4} \tau_{5} z_{2} z_{5}=0 \Longrightarrow a=-1 / \lambda^{2} \\
& \lambda z_{1} z_{6}+b \tau_{2} \tau_{3}^{2} \tau_{4}^{2} \tau_{5} z_{3} z_{4}=0 \Longrightarrow \quad b=-1 / \lambda^{3} \\
& \left.\begin{array}{r}
(\lambda-1) z_{1} z_{3}+b \tau_{4} \tau_{5}^{2} \tau_{6}^{2} \tau_{0} z_{5} z_{6}=0 \\
\tau_{1} z_{1}^{2}+b \tau_{3} \tau_{4}^{2} \tau_{5}^{2} \tau_{6} z_{4} z_{5}=0
\end{array}\right\} \Longrightarrow \lambda^{4}(\lambda-1)=\prod \tau_{i} .
\end{aligned}
$$

This completes the proof in the case $n=7$.

We proceed to study the schemes $T=T\left[\lambda ; \tau_{0}, \ldots, \tau_{n-1}\right]$. 
Lemma 2.9. Up to rescaling co-ordinates, the schemes $T\left[\lambda ; \tau_{0}, \ldots, \tau_{n-1}\right]$ over $K$ are uniquely determined by $\lambda \in K$ and $\theta=\prod \tau_{i}^{-i} \in K^{\times} /\left(K^{\times}\right)^{n}$.

Proof. Rescaling the co-ordinate $x_{1}$ by $u \in K^{\times}$gives

$$
T\left[\lambda ; \tau_{0}, \tau_{1}, \tau_{2}, \ldots, \tau_{n-1}\right] \cong T\left[\lambda ; u \tau_{0}, u^{-2} \tau_{1}, u \tau_{2}, \ldots, \tau_{n-1}\right] .
$$

Repeating for the other co-ordinates we find

$$
T\left[\lambda ; \tau_{0}, \tau_{1}, \tau_{2}, \ldots, \tau_{n-1}\right] \cong T\left[\lambda ; u^{-n} \tau_{0}, u^{n} \tau_{1}, \tau_{2}, \ldots, \tau_{n-1}\right] .
$$

The lemma follows easily from these observations.

An important consequence of Lemma 2.9 is that geometrically, i.e. over $\bar{K}$, the schemes $T=T\left[\lambda ; \tau_{0}, \ldots, \tau_{n-1}\right]$ are determined by $\lambda$.

Proposition 2.10. Let $\lambda, \tau_{0}, \ldots, \tau_{n-1} \in K$ with $\alpha(\lambda)=\prod \tau_{i} \neq 0$.

(i) If $\beta(\lambda) \neq 0$ then $T$ is a smooth curve of genus 1 .

(ii) If $\beta(\lambda)=0$ then $T$ is a Néron polygon.

Proof. By Lemma 2.9 we may suppose $K=\bar{K}$ and that $\tau_{0}=\tau_{1}=\ldots=\tau_{n-1}$. We apply the results of Sect. 2.1.

Case $n=5$. The curve defined by (17) is $T[\lambda ; \tau, \ldots, \tau]$ where $\tau=a / b$ and $\lambda=\tau^{5}$. Thus $T$ is either a smooth curve of genus 1 , or degenerates to a Néron polygon. The condition for degeneration is $\lambda=\phi^{5}$ or $\overline{\phi^{5}}$, equivalently $\lambda^{2}-11 \lambda-1=0$.

Case $n=7$. The curve defined by (18) is $T[\lambda ; \tau, \ldots, \tau]$ where $\lambda=-a c^{2} / b^{3}$ and $\tau=a c / b^{2}$. So the following birational map comes for free.

$$
\begin{aligned}
\left\{a^{3} b+b^{3} c+c^{3} a=0\right\} & \rightarrow\left\{\lambda^{4}(\lambda-1)=\tau^{7}\right\} \\
(a: b: c) & \mapsto\left(-a c^{2} / b^{3}, a c / b^{2}\right) \\
\left(\tau^{3}:-\lambda \tau: \lambda^{2}\right) & \leftrightarrow(\lambda, \tau)
\end{aligned}
$$

Again we deduce $T$ is either a smooth curve of genus 1 , or degenerates to a Néron polygon. The condition for degeneration is $\lambda=-\left(\zeta-\zeta^{6}\right)\left(\zeta^{4}-\zeta^{3}\right)^{2} /\left(\zeta^{2}-\zeta^{5}\right)^{3}$ or one of its conjugates, equivalently $\lambda^{3}-8 \lambda^{2}+5 \lambda+1=0$.

Lemma 2.11. The scheme $T\left[\lambda ; \tau_{0}, \ldots, \tau_{n-1}\right]$ meets the co-ordinate hyperplanes $H_{i}$ in a total of $n^{2}$ points. The points of intersection with $H_{i}$ are defined over $K\left(\zeta, \sqrt[n]{\alpha(\lambda)^{i} \theta}\right)$ where $\theta=\prod \tau_{i}^{-i}$.

Proof. Directly from the equations we may check $T \cap H_{i} \cap H_{j}=\emptyset$ for all $i \neq j$. Our first claim now follows from the above geometric description of $T$. For our second claim it suffices, by symmetry, to show that the points $T \cap H_{0}$ are defined over $K(\zeta, \sqrt[n]{\theta})$. But if $T=T[\lambda ; \alpha(\lambda), 1, \ldots, 1]$ then $T \cap H_{0}$ has a unique fixed point under

$$
[-1]:\left(x_{0}: x_{1}: \ldots: x_{n-1}\right) \mapsto\left(x_{0}: x_{n-1}: \ldots: x_{1}\right) .
$$

The uniqueness statement tells us that this point is $K$-rational. We are now done by Lemma 2.9 . 
We relate the curves $T\left[\lambda ; \tau_{0}, \ldots, \tau_{n-1}\right]$ to the torsors $C_{\lambda, \theta}$ defined in Sect. 1.2.

Proposition 2.12. Let $\lambda \in K$ not a cusp of $X_{1}(n)$.

(i) If $\tau_{0}, \ldots, \tau_{n-1} \in K$ with $\alpha(\lambda)=\prod \tau_{i}$ and $\theta=\prod \tau_{i}^{-i}$ then

$$
T\left[\lambda ; \tau_{0}, \ldots, \tau_{n-1}\right] \cong C_{\lambda, \theta}
$$

(ii) The image of $\mathbf{Z} / n \mathbf{Z} \hookrightarrow D_{\lambda}(K)$ under $\delta$ is generated by $\alpha(\lambda)$.

Proof. (i) We temporarily write $T_{\lambda}:=T[\lambda ; \alpha(\lambda), 1, \ldots, 1]$. By Lemma 2.11, $T_{\lambda}(K) \neq \varnothing$ and an explicit calculation reveals the rational point

$$
0=\left\{\begin{array}{l}
(0: 1: 1:-1:-1) \\
\left(0: 1:-\lambda:-\lambda^{2}: \lambda^{2}: \lambda:-1\right)
\end{array}\right.
$$

The case $\theta=1$ of the proposition is equivalent to the statement that the families of elliptic curves $C_{\lambda}$ and $T_{\lambda}$ are equal. So let $C / K$ be an elliptic curve with $\mu_{n} \hookrightarrow C$. We claim that $\mu_{n} \hookrightarrow C$ is isomorphic to $\mu_{n} \hookrightarrow T_{\lambda}$ for some unique $\lambda \in K$. We embed $C \hookrightarrow \mathbf{P}^{n-1}$ via the complete linear system $|n .0|$. Then by (15) and the argument at the end of Sect. 1.2, we may choose co-ordinates such that the action of $\mu_{n}$ on $C$ via translation is the diagonal action (22). We may further assume $0 \in C \cap\left\{x_{0}=0\right\}$. Now Proposition 2.8 tells us $C \cong T\left[\lambda ; \tau_{0}, \ldots, \tau_{n-1}\right]$ for some $\lambda, \tau_{0}, \ldots, \tau_{n-1} \in K$. By Lemma 2.11 we have $\theta=\prod \tau_{i}^{-i} \in\left(K^{\times}\right)^{n}$ and by Lemma 2.9 it follows $C \cong T_{\lambda}$. To check the uniqueness statement we consider the automorphisms of $\mathbf{P}^{n-1}$ that commute with (22). These are given by rescaling and cyclically permuting our co-ordinates. In particular they do not alter $\lambda$ and this proves our claim.

To show that the families $C_{\lambda}$ and $T_{\lambda}$ are equal, it only remains to check that we have chosen the same co-ordinate $\lambda$ on $X_{1}(n) \cong \mathbf{P}^{1}$ here as in Sect. 1.1. But in both cases our co-ordinate $\lambda$ has been chosen such that the cusps are as listed in Sect. 1.1. We have now shown $C_{\lambda}=T[\lambda ; \alpha(\lambda), 1, \ldots, 1]$.

Let $\tau_{0}, \ldots, \tau_{n-1}$ be as above. By Lemma 2.9 there is an isomorphism defined over $\bar{K}$

$$
\psi: T\left[\lambda ; \tau_{0}, \tau_{1}, \ldots, \tau_{n-1}\right] \stackrel{\sim}{\longrightarrow} C_{\lambda}=T[\lambda ; \alpha(\lambda), 1, \ldots, 1]
$$

given by rescaling co-ordinates. But the only automorphisms of $C_{\lambda}$ given by rescaling co-ordinates belong to our diagonal action of $\boldsymbol{\mu}_{n}$. Thus the cocycle $\sigma \mapsto \sigma(\psi) \psi^{-1}$ takes values in $\boldsymbol{\mu}_{n}$. We compute $\sigma(\psi) \psi^{-1}=\sigma(\sqrt[n]{\theta}) / \sqrt[n]{\theta}$.

(ii) Since

$$
T\left[\lambda ; \tau_{0}, \tau_{1}, \ldots, \tau_{n-1}\right] \cong T\left[\lambda ; \tau_{n-1}, \tau_{0}, \ldots, \tau_{n-2}\right]
$$

it is clear from (i) and the exact sequence (7) that $\alpha(\lambda) \in \operatorname{im} \delta$. Taking $\tau_{0}=\ldots=$ $\tau_{n-2}=1$ and $\tau_{n-1}=\alpha(\lambda)$ in (24) we construct $\psi: C_{\lambda} \rightarrow C_{\lambda}$, translation by an element of $C_{\lambda}[n]$, such that $\sigma(\psi) \psi^{-1}=\sigma(\sqrt[n]{\alpha(\lambda)}) / \sqrt[n]{\alpha(\lambda)}$. Thus $\alpha(\lambda) \in \operatorname{im} \delta$ is in fact the image of the torsion $\mathbf{Z} / n \mathbf{Z} \hookrightarrow D_{\lambda}(K)$. 
Remark 2.13. Relabelling our copy of $\boldsymbol{\mu}_{n}$ gives isomorphisms $C_{\lambda, \theta} \cong C_{-1 / \lambda, \theta^{2}}$ respectively $C_{\lambda, \theta} \cong C_{(\lambda-1) / \lambda, \theta^{2}}$. Proposition 2.12 allows us to explicitly write down these isomorphisms. For example in the case $n=5$ we have

$$
\begin{aligned}
& T\left[\lambda ; \tau_{0}, \tau_{1}, \tau_{2}, \tau_{3}, \tau_{4}\right] \cong T\left[-1 / \lambda ;-\tau_{0} /\left(\tau_{2} \tau_{3}\right), \ldots,-\tau_{3} /\left(\tau_{0} \tau_{1}\right)\right] \\
& \left(x_{0}: x_{1}: x_{2}: x_{3}: x_{4}\right) \mapsto\left(x_{0}: x_{2}: x_{4}: x_{1}: x_{3}\right) .
\end{aligned}
$$

Finally we drop our assumption $\operatorname{char}(K) \neq n$. The equations appearing in the statement of the next proposition are obtained from those for $T\left[\lambda ; \tau_{0}, \ldots, \tau_{n-1}\right]$ by manipulating under the assumption $\alpha(\lambda)=\prod \tau_{i} \neq 0$ and then setting $\lambda=0$, respectively $\lambda=1$. We write $P_{0}=(1: 0: 0: \ldots), P_{1}=(0: 1: 0: \ldots), \ldots$

Proposition 2.14. Let $\tau_{0}, \ldots, \tau_{n-1} \in K$ with $I=\left\{i \mid \tau_{i}=0\right\} \neq \emptyset$. Then the subscheme of $\mathbf{P}^{n-1}$ with equations

$$
\begin{array}{r}
n=5 \\
n=7\left\{\begin{aligned}
& \tau_{0} x_{0}^{2}+x_{1} x_{4}-\tau_{2} \tau_{3} x_{2} x_{3}=0 \text { \& cyclic permutes } \\
& \tau_{0} x_{0}^{2}+x_{1} x_{6}-\tau_{2} \tau_{3} \tau_{4} \tau_{5} x_{2} x_{5}=0 \\
& \tau_{0}^{2} \tau_{1} \tau_{6} x_{0}^{2}-x_{2} x_{5}+\tau_{3} \tau_{4} x_{3} x_{4}=0
\end{aligned}\right. \text { \& cyclic permutes }
\end{array}
$$

is the union of $|I|$ rational curves. More precisely for each consecutive pair $i, i+d \in I$ we have a rational curve of degree $d$ joining $P_{i}$ and $P_{i+d}$.

Proof. Rescaling co-ordinates, over $\bar{K}$ if necessary, we may suppose that the nonzero $\tau_{i}$ are all 1 . We split into cases according as the $\tau_{i}$ are 0 or 1 . In each case a straightforward calculation proves the proposition. Notice that in the most degenerate case, namely when all the $\tau_{i}$ are 0 , we obtain a Néron polygon.

\subsection{Local solubility for torsors}

In this section we work over a field which is complete with respect to a discrete valuation and has finite residue field. We use the results of Sect. 2.2 to give some criteria for the existence of rational points on the torsors $C_{\lambda, \theta}$. We adopt the following notation.

$$
\begin{array}{ll}
K & \text { a field complete with respect to the valuation ord }: K^{\times} \rightarrow \mathbf{Z} \\
\mathcal{O} & \text { the ring of integers of } K=\{x \in K \mid \operatorname{ord}(x) \geq 0\} \\
\mathfrak{p} & \text { the maximal ideal of } \mathcal{O}=\{x \in K \mid \operatorname{ord}(x)>0\} \\
k & \text { the residue field of } \mathcal{O}=\mathcal{O} / \mathfrak{p} \text { (assumed finite). }
\end{array}
$$

We define $\operatorname{ord}^{*}(\lambda)$ to be the non-negative integer $r$ with

$$
\begin{array}{lll}
n=5 & \{\operatorname{ord}(\lambda), \operatorname{ord}(-1 / \lambda)\} & =\{-r, r\} \\
n=7 & \{\operatorname{ord}(\lambda), \operatorname{ord}((\lambda-1) / \lambda), \operatorname{ord}(1 /(1-\lambda))\} & =\{-r, 0, r\} .
\end{array}
$$

Proposition 2.15. Let $\lambda \in K$ not a cusp of $X_{1}(n)$. We describe the image of the connecting homomorphism $\delta: D_{\lambda}(K) \rightarrow K^{\times} /\left(K^{\times}\right)^{n}$.

(a) If $\operatorname{ord}^{*}(\lambda)>0$ then $\operatorname{im} \delta=K^{\times} /\left(K^{\times}\right)^{n}$.

(b) If $\operatorname{ord}^{*}(\lambda)=0$ then $\operatorname{im} \delta \subset \mathcal{O}^{\times} /\left(\mathcal{O}^{\times}\right)^{n}$.

(c) If $\operatorname{ord}^{*}(\lambda)=0$ and $\operatorname{char}(k) \neq n$ then

(i) If $\beta(\lambda) \not \equiv 0(\bmod \mathfrak{p})$ then $\operatorname{im} \delta=\mathcal{O}^{\times} /\left(\mathcal{O}^{\times}\right)^{n}$.

(ii) If $\beta(\lambda) \equiv 0(\bmod \mathfrak{p})$ then $\operatorname{im} \delta=0$. 
Proof. Let us note that by the work of Sect. 1.2, $C_{\lambda, \theta}(K) \neq \emptyset \Longleftrightarrow \theta \in \operatorname{im} \delta$. (a) Let $\theta \in K^{\times} /\left(K^{\times}\right)^{n}$. By Remark 2.13 we may assume $\operatorname{ord}(\lambda)>0$, respectively $\operatorname{ord}(\lambda-1)>0$. We write $C_{\lambda, \theta}$ in the form $T\left[\lambda ; \tau_{0}, \ldots, \tau_{n-1}\right]$ with $\operatorname{ord}\left(\tau_{i}\right) \geq 0$ for all $i$. By Proposition 2.14 the reduction of this curve is a collection of rational curves each defined over $k$. At least one of these curves has odd degree and so is isomorphic to $\mathbf{P}^{1}$ over $k$. We pick a smooth point on the reduction and lift using Hensel's lemma to give $C_{\lambda, \theta}(K) \neq \emptyset$ as required.

(b) Suppose $\theta \in K^{\times} /\left(K^{\times}\right)^{n}$ with $\operatorname{ord}(\theta) \equiv 1(\bmod n)$. We write $C_{\lambda, \theta}$ in the form $T\left[\lambda ; \tau_{0}, \ldots, \tau_{n-1}\right]$ with $\operatorname{ord}\left(\tau_{0}\right)=-1, \operatorname{ord}\left(\tau_{n-1}\right)=1$ and $\operatorname{ord}\left(\tau_{i}\right)=0$ for all other $i$. We take $\left(x_{0}: \ldots: x_{n-1}\right)$ a $K$-point with $\min \left\{\operatorname{ord}\left(x_{i}\right)\right\}=0$. Examining the equations for $T\left[\lambda ; \tau_{0}, \ldots, \tau_{n-1}\right]$ we successively deduce $\operatorname{ord}\left(x_{i}\right)>0$ for $i=$ $0,1, \ldots, n-1$. This contradiction tells us $C_{\lambda, \theta}(K)=\emptyset$. Thus $\operatorname{im} \delta \subset \mathcal{O}^{\times} /\left(\mathcal{O}^{\times}\right)^{n}$. (c) Let $\theta \in \mathcal{O}^{\times} /\left(\mathcal{O}^{\times}\right)^{n}$ and write $C_{\lambda, \theta}$ in the form $T\left[\lambda ; \tau_{0}, \ldots, \tau_{n-1}\right]$ with $\operatorname{ord}\left(\tau_{i}\right)=0$ for all $i$. The reduction of this curve is described by Proposition 2.10. In case (i) the reduction is a smooth curve of genus 1 . By [Ca3, Chapter 25] we have a rational point which we lift using Hensel's lemma. Thus im $\delta=\mathcal{O}^{\times} /\left(\mathcal{O}^{\times}\right)^{n}$ as required. In case (ii) the reduction is a collection of $n$ distinct lines. If $C_{\lambda, \theta}(K) \neq \emptyset$ then one of these lines must be defined over $k$. This line meets each of the hyperplanes $H_{i}$. By Lemma 2.11 we deduce that both $\alpha(\lambda)$ and $\theta$ are $n$th powers $\bmod \mathfrak{p}$. Since $\operatorname{char}(k) \neq n$ it follows $\theta \in\left(K^{\times}\right)^{n}$. Thus $\operatorname{im} \delta=0$ as required.

Proposition 2.15 is incomplete in that we do not fully treat the case $\operatorname{char}(k)=n$. In particular, if $\beta(\lambda) \equiv 0(\bmod \mathfrak{p})$, then the equations for $T\left[\lambda ; \tau_{0}, \ldots, \tau_{n-1}\right]$ reduce to give a line with multiplicity $n$. This makes it difficult to apply Hensel's lemma. We therefore abandon the projective space method and revert to using the push-out method also described in Sect. 1.2. Specifically we recall that the map

$$
\delta: D_{\lambda}(K) \rightarrow K^{\times} /\left(K^{\times}\right)^{n}
$$

may be described in terms of a rational function $f \in K\left(D_{\lambda}\right)$, explicitly given by (9). We use the formal group defined by $D_{\lambda}$ as a vehicle for our Hensel lemma calculations. We recall [Si1, Chapter IV] that the Weierstrass equation

$$
y^{2}+a_{1} x y+a_{3} y=x^{3}+a_{2} x^{2}+a_{4} x+a_{6}
$$

is satisfied by the power series

$$
\begin{aligned}
& x(z)=\frac{1}{z^{2}}-\frac{a_{1}}{z}-a_{2}-a_{3} z-\left(a_{4}+a_{1} a_{3}\right) z^{2}-\ldots \\
& y(z)=-\frac{1}{z^{3}}+\frac{a_{1}}{z^{2}}+\frac{a_{2}}{z}+a_{3}+\left(a_{4}+a_{1} a_{3}\right) z+\ldots
\end{aligned}
$$

Applying this to the curves $D_{\lambda}$ defined by (2) we find that $x(z)$ and $y(z)$ are power series with coefficients in $\mathbf{Z}[\lambda]$. We compute the first few terms of the power series $F(z):= \pm z^{n} f(x(z), y(z))$.

$$
\begin{aligned}
& F(z)=1+(2 \lambda-1) z+\lambda(\lambda+2) z^{2}+\ldots \\
& F(z)=1+\left(3 \lambda^{2}-2 \lambda-2\right) z+(\lambda-1)\left(3 \lambda^{3}+3 \lambda^{2}-3 \lambda-1\right) z^{2}+\ldots
\end{aligned}
$$


Our interest is in the case $\operatorname{char}(k)=n$. We find $\beta(\lambda) \equiv(\lambda-3)^{2}(\bmod \mathfrak{p})$, respectively $\beta(\lambda) \equiv(\lambda-5)^{3}(\bmod \mathfrak{p})$, and $F^{\prime}(0) \equiv \beta^{\prime}(\lambda)(\bmod \mathfrak{p})$. Thus

$$
\begin{array}{lll}
F(\mathfrak{p O})=1+\mathfrak{p O} & \text { if } \beta(\lambda) \not \equiv 0 & (\bmod \mathfrak{p}) \\
F(\mathfrak{p O}) \subset 1+\mathfrak{p}^{2} \mathcal{O} & \text { if } \beta(\lambda) \equiv 0 & (\bmod \mathfrak{p}) .
\end{array}
$$

In the case $K=\mathbf{Q}_{p}$ this gives us all we need.

Proposition 2.16. Let $p=n$. Let $\lambda \in \mathbf{Q}_{p}$ not a cusp of $X_{1}(n)$. We describe the image of $\delta: D_{\lambda}\left(\mathbf{Q}_{p}\right) \rightarrow \mathbf{Q}_{p}^{\times} /\left(\mathbf{Q}_{p}^{\times}\right)^{n}$ in the case $\operatorname{ord}^{*}(\lambda)=0$.

(i) If $\beta(\lambda) \not \equiv 0(\bmod p)$ then $\operatorname{im} \delta=\mathbf{Z}_{p}^{\times} /\left(\mathbf{Z}_{p}^{\times}\right)^{n}$.

(ii) If $\beta(\lambda) \equiv 0(\bmod p)$ then $\operatorname{im} \delta$ is generated by $\alpha(\lambda)$. Thus the condition for $\delta$ to be the zero map is $\lambda \equiv 18(\bmod 25)$, respectively $\lambda \equiv 5(\bmod 7)$.

Proof. As observed in Sect. 1.1, the Weierstrass equation (2) is minimal. We write $E=D_{\lambda}$ and recall [Si1, Chapter VII] there is a filtration

$$
E\left(\mathbf{Q}_{p}\right) \supset E_{0}\left(\mathbf{Q}_{p}\right) \supset E_{1}\left(\mathbf{Q}_{p}\right) .
$$

By Lemma 1.4 the image of $\delta$ restricted to $E_{1}\left(\mathbf{Q}_{p}\right)$ is generated by $F\left(p \mathbf{Z}_{p}\right)$.

(i) By Proposition 2.15(b) we have im $\delta \subset \mathbf{Z}_{p}^{\times} /\left(\mathbf{Z}_{p}^{\times}\right)^{n}$. Since $1+p \mathbf{Z}_{p}$ generates $\mathbf{Z}_{p}^{\times} /\left(\mathbf{Z}_{p}^{\times}\right)^{n}$ it follows from (25) that we have equality.

(ii) Since $1+p^{2} \mathbf{Z}_{p} \subset\left(\mathbf{Z}_{p}^{\times}\right)^{n}$, it follows from (26) that $\delta$ restricted to $E_{1}\left(\mathbf{Q}_{p}\right)$ is trivial. Next $E_{0}\left(\mathbf{Q}_{p}\right) / E_{1}\left(\mathbf{Q}_{p}\right) \cong \widetilde{E}_{\text {ns }}(\mathbf{Z} / n \mathbf{Z}) \cong \mathbf{Z} / n \mathbf{Z}$, so by Proposition 2.12(ii) the image of $\delta$ restricted to $E_{0}\left(\mathbf{Q}_{p}\right)$ is generated by $\alpha(\lambda)$. Finally in the case of additive reduction, the Tamagawa number $\left[E\left(\mathbf{Q}_{p}\right): E_{0}\left(\mathbf{Q}_{p}\right)\right]$ is at most 4 , and so prime to $n$. It follows that there is no further contribution to the image of $\delta$. For the last statement we compute

$$
\begin{gathered}
\left\{\begin{array}{c}
\lambda \equiv 3(\bmod 5) \\
\lambda \in\left(\mathbf{Q}_{5}^{\times}\right)^{5}
\end{array}\right\} \Longleftrightarrow \lambda \equiv 18(\bmod 25) \\
\left\{\begin{array}{c}
\lambda \equiv 5(\bmod 7) \\
\lambda^{4}(\lambda-1) \in\left(\mathbf{Q}_{7}^{\times}\right)^{7}
\end{array}\right\} \Longleftrightarrow \lambda \equiv 5(\bmod 7) .
\end{gathered}
$$

\subsection{Proof of the descent theorem}

Let $n=5$ or 7. Let $\lambda \in \mathbf{Q}$ not a cusp of $X_{1}(n)$ and let $\mathcal{A}, \mathcal{B}$ be the sets of rational primes defined in Sect. 1.3. In this section we prove that the Selmer groups $S^{(\phi)}\left(C_{\lambda} / \mathbf{Q}\right)$ and $S^{(\widehat{\phi})}\left(D_{\lambda} / \mathbf{Q}\right)$ are as described in Theorem 1.

Let $p$ be a rational prime. The Tate pairing

$$
(\cdot, \cdot)_{p}: H^{1}\left(\mathbf{Q}_{p}, C[\phi]\right) \times H^{1}\left(\mathbf{Q}_{p}, D[\widehat{\phi}]\right) \rightarrow \mathbf{Q} / \mathbf{Z}
$$

is induced by cup product from the Weil pairing, followed by the "invariant" map $\operatorname{Br}\left(\mathbf{Q}_{p}\right) \rightarrow \mathbf{Q} / \mathbf{Z}$. Since $C[\phi] \cong \boldsymbol{\mu}_{n}$ and $D[\widehat{\phi}] \cong \mathbf{Z} / n \mathbf{Z}$ we identify $H^{1}\left(\mathbf{Q}_{p}, C[\phi]\right)=$ $\mathbf{Q}_{p}^{\times} /\left(\mathbf{Q}_{p}^{\times}\right)^{n}$ and $H^{1}\left(\mathbf{Q}_{p}, D[\widehat{\phi}]\right)=\operatorname{Hom}\left(G_{p}, \mathbf{Z} / n \mathbf{Z}\right)$. Here our notation is $G_{p}:=$ $\operatorname{Gal}\left(\overline{\mathbf{Q}}_{p} / \mathbf{Q}_{p}\right)$ and we write $I_{p} \subset G_{p}$ for the inertia subgroup. 
Proposition 2.17. Let $\lambda \in \mathbf{Q}$ not a cusp of $X_{1}(n)$. The connecting maps

$$
\begin{aligned}
& \delta_{\phi}: D_{\lambda}\left(\mathbf{Q}_{p}\right) \rightarrow H^{1}\left(\mathbf{Q}_{p}, C_{\lambda}[\phi]\right) \\
& \delta_{\widehat{\phi}}: C_{\lambda}\left(\mathbf{Q}_{p}\right) \rightarrow H^{1}\left(\mathbf{Q}_{p}, D_{\lambda}[\widehat{\phi}]\right)
\end{aligned}
$$

have images

$$
\operatorname{im} \delta_{\phi}= \begin{cases}\mathbf{Q}_{p}^{\times} /\left(\mathbf{Q}_{p}^{\times}\right)^{n} & \text { if } p \in \mathcal{A} \\ \mathbf{Z}_{p}^{\times} /\left(\mathbf{Z}_{p}^{\times}\right)^{n} & \text { if } p \notin \mathcal{A} \cup \mathcal{B} \\ 0 & \text { if } p \in \mathcal{B}\end{cases}
$$

and

$$
\operatorname{im} \delta_{\widehat{\phi}}= \begin{cases}0 & \text { if } p \in \mathcal{A} \\ \operatorname{Hom}\left(G_{p} / I_{p}, \mathbf{Z} / n \mathbf{Z}\right) & \text { if } p \notin \mathcal{A} \cup \mathcal{B} \\ \operatorname{Hom}\left(G_{p}, \mathbf{Z} / n \mathbf{Z}\right) & \text { if } p \in \mathcal{B} .\end{cases}
$$

Proof. (i) The description of $\operatorname{im} \delta_{\phi}$ is given by combining Propositions 2.15 and 2.16. Note that $\mathbf{Z}_{p}^{\times} /\left(\mathbf{Z}_{p}^{\times}\right)^{n}$ is trivial unless $p=n$ or $p \equiv 1(\bmod n)$.

(ii) Tate local duality [T1, Theorem 2.3] tells us that for $E$ an elliptic curve over $\mathbf{Q}_{p}$ there is a non-degenerate pairing $E\left(\mathbf{Q}_{p}\right) \times H^{1}\left(\mathbf{Q}_{p}, E\right) \rightarrow \mathbf{Q} / \mathbf{Z}$ compatible with the Tate pairing (27). We obtain a commutative diagram with exact rows

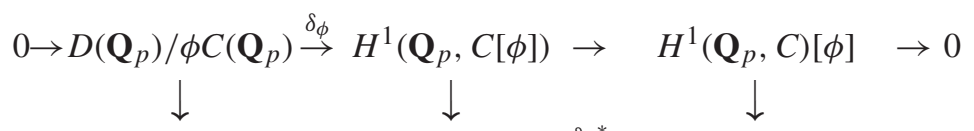

$$
\begin{aligned}
& 0 \rightarrow H^{1}\left(\mathbf{Q}_{p}, D\right)[\widehat{\phi}]^{*} \rightarrow H^{1}\left(\mathbf{Q}_{p}, D[\widehat{\phi}]\right)^{*} \stackrel{\delta_{\widehat{\phi}^{*}}^{*}}{\rightarrow}\left(C\left(\mathbf{Q}_{p}\right) / \widehat{\phi} D\left(\mathbf{Q}_{p}\right)\right)^{*} \rightarrow 0
\end{aligned}
$$

Since the vertical maps are isomorphisms we learn that $\operatorname{im} \delta_{\phi}$ and $\operatorname{im} \delta_{\widehat{\phi}}$ are exact annihilators with respect to the Tate pairing. Thus the description of im $\delta_{\widehat{\phi}}$ follows from the description of $\operatorname{im} \delta_{\phi}$. It remains to check that $\mathbf{Z}_{p}^{\times} /\left(\mathbf{Z}_{p}^{\times}\right)^{n}$ and $\operatorname{Hom}\left(G_{p} / I_{p}, \mathbf{Z} / n \mathbf{Z}\right)$ are exact annihilators. We recall [Se2, Chapter XIV] that the Tate pairing

$$
\mathbf{Q}_{p}^{\times} /\left(\mathbf{Q}_{p}^{\times}\right)^{n} \times \operatorname{Hom}\left(G_{p}, \mathbf{Z} / n \mathbf{Z}\right) \rightarrow \mathbf{Z} / n \mathbf{Z}
$$

is given by $(\theta, \chi) \mapsto-\chi(\rho(\theta))$ where $\rho: \mathbf{Q}_{p}^{\times} \rightarrow G_{p}^{\text {ab }}$ is the local reciprocity map. Thus our claim follows from the description of the local reciprocity map in the unramified case [CF, Chapter VI, §2.5].

The description of $S^{(\phi)}\left(C_{\lambda} / \mathbf{Q}\right)$ given in Theorem 1 is immediate from Proposition 2.17(i).

$$
\begin{aligned}
S^{(\phi)}\left(C_{\lambda} / \mathbf{Q}\right) & =\left\{\theta \in \mathbf{Q}^{\times} /\left(\mathbf{Q}^{\times}\right)^{n} \mid C_{\lambda, \theta}\left(\mathbf{Q}_{p}\right) \neq \emptyset \text { for all primes } p\right\} \\
& =\left\{\theta \in[\mathcal{A}] \mid \theta \in\left(\mathbf{Q}_{p}^{\times}\right)^{n} \text { for all } p \in \mathcal{B}\right\} .
\end{aligned}
$$

We have already seen two proofs of the statement concerning torsion, in Sect. 1.2 via the push-out method, and in Sect. 2.2 via the projective space method. So to prove Theorem 1 it only remains to explain the relationship between $S^{(\phi)}\left(C_{\lambda} / \mathbf{Q}\right)$ and $S^{(\widehat{\phi})}\left(D_{\lambda} / \mathbf{Q}\right)$. We shall make use of the "product" formula for the local Tate 
pairings, namely

$$
\sum_{p}(a, b)_{p}=0
$$

This is a consequence of the well known exact sequence of class field theory

$$
0 \longrightarrow \operatorname{Br}(\mathbf{Q}) \longrightarrow \oplus_{v} \operatorname{Br}\left(\mathbf{Q}_{v}\right) \stackrel{\sum \text { inv }}{\longrightarrow} \mathbf{Q} / \mathbf{Z} \longrightarrow 0
$$

where again we have used the fact $n$ is odd to ignore the infinite places. For $\mathcal{S}$ a finite set of rational primes we write

$$
\begin{aligned}
& {[\mathcal{S}]:=\left\{\theta \in \mathbf{Q}^{\times} /\left(\mathbf{Q}^{\times}\right)^{n} \mid \operatorname{ord}_{p}(\theta) \equiv 0(\bmod n) \text { for all } p \notin \mathcal{S}\right\}} \\
& \langle\mathcal{S}\rangle:=\left\{\chi \in \operatorname{Hom}\left(G_{\mathbf{Q}}, \mathbf{Z} / n \mathbf{Z}\right) \mid \chi\left(I_{p}\right)=0 \text { for all } p \notin \mathcal{S}\right\}
\end{aligned}
$$

We define $\Xi:[\mathcal{A}] \times\langle\mathcal{B}\rangle \rightarrow \mathbf{Z} / n \mathbf{Z}$ via

$$
\Xi(a, b)=\sum_{p \in \mathcal{B}}(a, b)_{p}=-\sum_{p \in \mathcal{A}}(a, b)_{p}
$$

where the two expressions given are equal by Proposition 2.17 and the product formula. Since $\mathcal{B}$ consists of primes $p \equiv 0,1(\bmod n)$ it follows by class field theory, specifically the Kronecker-Weber theorem, that $\langle\mathcal{B}\rangle$ has dimension $|\mathcal{B}|$. Thus $[\mathcal{B}] \cong\langle\mathcal{B}\rangle$ as $\mathbf{Z} / n \mathbf{Z}$-vector spaces. So to complete the proof of Theorem 1 it only remains to identify the left and right kernels of $\Xi$ as Selmer groups. For any element $(*)$ we write $(*)_{p}$ for the corresponding local element.

$$
\begin{aligned}
S^{(\phi)}\left(C_{\lambda} / \mathbf{Q}\right) & =\left\{a \in \mathbf{Q}^{\times} /\left(\mathbf{Q}^{\times}\right)^{n} \mid a_{p} \in \operatorname{im} \delta_{\phi} \text { for all primes } p\right\} \\
& =\left\{a \in[\mathcal{A}] \mid a_{p}=0 \text { for all } p \in \mathcal{B}\right\} \\
& \subset \operatorname{ker}_{L}(\Xi) \\
S^{(\widehat{\phi})}\left(D_{\lambda} / \mathbf{Q}\right) & =\left\{b \in \operatorname{Hom}\left(G_{\mathbf{Q}}, \mathbf{Z} / n \mathbf{Z}\right) \mid b_{p} \in \operatorname{im} \delta_{\widehat{\phi}} \text { for all primes } p\right\} \\
& =\left\{b \in\langle\mathcal{B}\rangle \mid b_{p}=0 \text { for all } p \in \mathcal{A}\right\} \\
& \subset \operatorname{ker}_{R}(\Xi) .
\end{aligned}
$$

To show that these inclusions are in fact equalities we need a lemma.

Lemma 2.18. The following natural maps are isomorphisms.

(i) $[\mathcal{A}] \rightarrow \oplus_{p \in \mathcal{A}} \mathbf{Q}_{p}^{\times} /\left(\mathbf{Q}_{p}^{\times}\right)^{n} / \mathbf{Z}_{p}^{\times} /\left(\mathbf{Z}_{p}^{\times}\right)^{n}$

(ii) $\langle\mathcal{B}\rangle \rightarrow \oplus_{p \in \mathcal{B}} \operatorname{Hom}\left(G_{p}, \mathbf{Z} / n \mathbf{Z}\right) / \operatorname{Hom}\left(G_{p} / I_{p}, \mathbf{Z} / n \mathbf{Z}\right)$

Proof. (i) The map is injective since

$$
\left\{\theta \in \mathbf{Q}^{\times} /\left(\mathbf{Q}^{\times}\right)^{n} \mid \operatorname{ord}_{p}(\theta) \equiv 0(\bmod n) \text { for all } p\right\}
$$

is trivial. Both spaces have dimension $|\mathcal{A}|$.

(ii) The map is injective since $\mathbf{Q}$ has no unramified (abelian) extensions. For $p \equiv 0,1(\bmod n)$ we know $\mathbf{Z}_{p}^{\times} /\left(\mathbf{Z}_{p}^{\times}\right)^{n}$ is cyclic of order $n$. Thus both spaces have dimension $|\mathcal{B}|$. 
Theorem 1 now follows by (28) and the exact annihilation of $\mathbf{Z}_{p}^{\times} /\left(\mathbf{Z}_{p}^{\times}\right)^{n}$ and $\operatorname{Hom}\left(G_{p} / I_{p}, \mathbf{Z} / n \mathbf{Z}\right)$.

\section{Examples and applications}

\subsection{Relationship with the parity conjecture}

The estimate (13) for $r=\operatorname{rank} C_{\lambda}(\mathbf{Q})=\operatorname{rank} D_{\lambda}(\mathbf{Q})$ differs from the true rank by

$$
\operatorname{dim}_{n} \amalg\left(C_{\lambda} / \mathbf{Q}\right)[\phi]+\operatorname{dim}_{n} \amalg\left(D_{\lambda} / \mathbf{Q}\right)[\widehat{\phi}]
$$

Let us assume that $\amalg\left(C_{\lambda} / \mathbf{Q}\right)(n)$ and $\amalg\left(D_{\lambda} / \mathbf{Q}\right)(n)$ are both finite. Since the maps $\phi$ and $\widehat{\phi}$ on Tate-Shafarevich groups are adjoints with respect to the Cassels-Tate pairing [Ca2, Theorem 1.2], it follows that (29) is even. So Theorem 1 furnishes the following parity result.

Corollary 1. Let $E / \mathbf{Q}$ be an elliptic curve with a rational point of order $n=5$ or 7. Assume $\amalg(E / \mathbf{Q})(n)$ is finite. Then the parity of the rank of $E(\mathbf{Q})$ is as predicted by the Birch Swinnerton-Dyer conjecture.

Proof. Theorem 1 and the finiteness assumption on II tells us

$$
\operatorname{rank} E(\mathbf{Q}) \equiv|\mathcal{A}|+|\mathcal{B}|-1 \quad(\bmod 2) .
$$

On the other hand, the Birch Swinnerton-Dyer conjecture predicts

$$
(-1)^{\operatorname{rank} E(\mathbf{Q})}=\prod_{v} W_{v}
$$

where $W_{v}= \pm 1$ are the local root numbers. Since $W_{\infty}=-1$ for any elliptic curve over $\mathbf{Q}$ it suffices for us to prove

$$
\left\{p \text { prime } \mid W_{p}=-1\right\}=\mathcal{A} \cup \mathcal{B} .
$$

The following explicit description of the local root numbers for an elliptic curve over $\mathbf{Q}$ is taken from [R]. See also [Co].

Proposition 3.1. Let $E$ be an elliptic curve over $\mathbf{Q}$ and let $p$ be a prime.

(i) If $E$ has good reduction at $p$ then $W_{p}=+1$.

(ii) If $E$ has multiplicative reduction at $p$ then

$$
W_{p}= \begin{cases}-1 & \text { if } E \text { has split reduction } \\ +1 & \text { if } E \text { has non-split reduction. }\end{cases}
$$

(iii) If $E$ has additive reduction at $p$ and $p \geq 5$ then

$$
W_{p}= \begin{cases}(-1 / p) & \text { if } e=2 \text { or } 6 \\ (-3 / p) & \text { if } e=3 \\ (-2 / p) & \text { if } e=4\end{cases}
$$

where $(a / p)$ is the quadratic Legendre symbol and $e=12 / \operatorname{gcd}\left(\operatorname{ord}_{p}(\Delta), 12\right)$. 
Our claim (30) now follows from the description of the reduction types in Lemma 1.3. The following table summarises the calculations required in the case of additive reduction.

\begin{tabular}{cc|ccc}
$p=n$ & $\lambda$ & $\operatorname{ord}_{p}(\beta(\lambda))$ & $e$ & $W_{p}$ \\
\hline 5 & $\operatorname{ord}_{5}(\lambda-18)=1$ & 2 & 6 & +1 \\
5 & $\operatorname{ord}_{5}(\lambda-18) \geq 2$ & 3 & 4 & -1 \\
7 & $\operatorname{ord}_{7}(\lambda-5) \geq 1$ & 2 & 6 & -1
\end{tabular}

Remark 3.2. Corollary 1 is in fact a special case of a result of Monsky [Mo], who works with elliptic curves $E$ over $\mathbf{Q}$ admitting a rational isogeny of prime degree. His method is to find a quadratic twist of $E$ with analytic rank 0 , and then to apply the work of Kolyvagin and others.

\subsection{Exhibiting large selmer groups}

We may use Theorem 1 to exhibit elliptic curves with large $n$-Selmer group. To do this we choose $\lambda \in \mathbf{Q}$ such that $|\mathcal{A}|$ is large and $|\mathcal{B}|$ is small, or vice versa. I am grateful to Ed Schaefer for bringing to my attention the following result.

Proposition 3.3. Let $F(x)$ be an irreducible polynomial of degree $d$ with integer coefficients. Suppose that for all primes $p$ the congruence $F(x) \equiv 0(\bmod p)$ has fewer than $p$ solutions. Then there exist infinitely many integers $y$ such that $F(y)$ consists of at most $d+1$ prime factors.

Proof. [HR, Theorem 9.7].

Corollary 2. For $n=5$ or 7 the $n$-Selmer group of an elliptic curve over $\mathbf{Q}$ may become arbitrarily large.

Proof. Let $M$ be the product of the first $m$ primes. Applying Proposition 3.3 to the polynomial $F(x)=\beta(M x)$ gives infinitely many integers $\lambda=M y$ with $|\mathcal{A}| \geq m$ and $|\mathcal{B}| \leq(n+1) / 2$. The corollary is now immediate from Theorem 1 .

We could equally prove Corollary 2 by taking $|\mathcal{A}|$ small and $|\mathcal{B}|$ large. Lemma 1.8 shows how we may force $|\mathcal{B}|$ to be large by imposing linear congruences on $\lambda$. In the case $n=5$ we then appeal to Dirichlet's theorem on primes in arithmetic progression to give $|\mathcal{A}|=1$. (In fact this is all we shall need in the next section to show that the 5-torsion of the Tate-Shafarevich group may become arbitrarily large.) In the case $n=7$ we appeal to [HR, Theorem 10.11] which generalises Proposition 3.3 to $F(x)$ a product of distinct irreducible polynomials. It seems we must take $\lambda=$ (integer) $/ 2$ in order to satisfy the hypothesis at $p=2$. 


\subsection{Exhibiting large Tate-Shafarevich groups}

Results of Cassels [Ca1], Bölling [Bö] and Kramer [Kr] show that the 2- and 3-torsion of the Tate-Shafarevich group of an elliptic curve over $\mathbf{Q}$ may become arbitrarily large. In this section we give a corresponding result for the 5-torsion. The idea is that the elliptic curves $E / \mathbf{Q}$ with $E[5] \cong \boldsymbol{\mu}_{5} \oplus \mathbf{Z} / 5 \mathbf{Z}$ belong to both the families $C_{\lambda}$ and $D_{\lambda}$. We may therefore estimate rank $E(\mathbf{Q})$ by applying Theorem 1 for two different values of $\lambda$. We then arrange for these estimates to differ.

Corollary 3. The 5-torsion of the Tate-Shafarevich group of an elliptic curve over $\mathbf{Q}$ may become arbitrarily large.

We define polynomials

$$
\begin{aligned}
& f(\tau)=\tau^{4}+3 \tau^{3}+4 \tau^{2}+2 \tau+1=\left((\tau+1)^{5}+1\right) /(\tau+2) \\
& g(\tau)=\tau^{4}-2 \tau^{3}+4 \tau^{2}-3 \tau+1=\left((\tau-1)^{5}+\tau^{5}\right) /(2 \tau-1) .
\end{aligned}
$$

Lemma 3.4. The elliptic curves $E / \mathbf{Q}$ with $E[5] \cong \mu_{5} \oplus \mathbf{Z} / 5 \mathbf{Z}$ are the curves $E_{\tau}:=C_{\tau^{5}} \cong D_{\tau f(\tau) / g(\tau)}$ for $\tau \in \mathbf{Q}$ with $\tau \neq 0$.

Proof. The statement concerning torsion in Theorem 1 tells us that the required universal family is $E_{\tau}:=C_{\tau^{5}}$. Here $\tau$ is a co-ordinate on $X(5) \cong \mathbf{P}^{1}$ and the cusps are at $\tau=0, \infty, \zeta^{i} \phi, \zeta^{i} \bar{\phi}$, where $\phi=1+\zeta+\zeta^{4}$ is the golden ratio and $\bar{\phi}=1+\zeta^{2}+\zeta^{3}$ is its conjugate. There are involutions

$$
\begin{aligned}
\eta: X_{1}(5) & \rightarrow X_{1}(5) ; & \lambda & \mapsto\left(\phi^{5} \lambda+1\right) /\left(\lambda-\phi^{5}\right) \\
\varepsilon: X(5) & \rightarrow X(5) ; & \tau & \mapsto(\phi \tau+1) /(\tau-\phi)
\end{aligned}
$$

mapping cusps to cusps. In fact $C_{\lambda} \cong D_{\eta(\lambda)}$ over $\mathbf{Q}\left(\boldsymbol{\mu}_{5}\right)$, whereas $\varepsilon$ belongs to the action of $\mathrm{PSL}_{2}(\mathbf{Z} / 5 \mathbf{Z})$ on $X(5)$. The following isomorphisms are defined over $\mathbf{Q}\left(\mu_{5}\right)$

$$
C_{\tau^{5}} \cong E_{\tau} \cong E_{\varepsilon(\tau)} \cong C_{\varepsilon(\tau)^{5}} \cong D_{\eta\left(\varepsilon(\tau)^{5}\right)} .
$$

We find $\eta\left(\varepsilon(\tau)^{5}\right)=\tau f(\tau) / g(\tau)$ and the isomorphism (31) maps $\mathbf{Z} / 5 \mathbf{Z} \hookrightarrow C_{\tau^{5}}$ onto $\mathbf{Z} / 5 \mathbf{Z} \hookrightarrow D_{\tau f(\tau) / g(\tau)}$. Comparing this isomorphism with its Galois conjugates, and appealing to Lemma 1.1(iii), we deduce $C_{\tau^{5}} \cong D_{\tau f(\tau) / g(\tau)}$ over $\mathbf{Q}$.

For $\tau \in \mathbf{Q}$ with $\tau \neq 0$ we define disjoint sets of rational primes

$$
\begin{aligned}
& \mathcal{P}=\left\{p \text { prime } \mid \operatorname{ord}_{p}(\tau) \neq 0\right\} \\
& \mathcal{Q}=\{p \text { prime } \mid f(\tau) g(\tau) \equiv 0(\bmod p) \text { and } p \equiv 1(\bmod 5)\} \\
& \mathcal{R}=\left\{p \text { prime } \mid \tau^{2}-\tau-1 \equiv 0(\bmod p) \text { and } p \equiv 0,1(\bmod 5)\right\} .
\end{aligned}
$$

Lemma 3.5. For $p$ a rational prime,

(i) $f(\tau) \equiv 0(\bmod p)$ is soluble $\Longleftrightarrow p=5$ or $p \equiv 1(\bmod 5)$

(ii) $g(\tau) \equiv 0(\bmod p)$ is soluble $\Longleftrightarrow p=5$ or $p \equiv 1(\bmod 5)$

(iii) $\tau^{2}-\tau-1 \equiv 0(\bmod p)$ is soluble $\Longleftrightarrow p=5$ or $p \equiv \pm 1(\bmod 5)$.

Proof. Consider how $p$ factors in $\mathbf{Q}\left(\boldsymbol{\mu}_{5}\right)$. 
Lemma 3.6. We apply Theorem 1 for $\lambda=\tau^{5}$ and $\lambda=\tau f(\tau) / g(\tau)$.

(i) If $\lambda=\tau^{5}$ then $\mathcal{A}=\mathcal{P}$ and $\mathcal{B}=\mathcal{Q} \cup \mathcal{R}$.

(ii) If $\lambda=\tau f(\tau) / g(\tau)$ then $\mathcal{A}=\mathcal{P} \cup \mathcal{Q}$ and $\mathcal{B}=\mathcal{R}$.

Proof. These claims follow from the identities

$$
\begin{aligned}
\tau^{10}-11 \tau^{5}-1 & =\left(\tau^{2}-\tau-1\right) f(\tau) g(\tau) \\
\tau^{2} f(\tau)^{2}-11 \tau f(\tau) g(\tau)-g(\tau)^{2} & =\left(\tau^{2}-\tau-1\right)^{5} .
\end{aligned}
$$

For $\tau$ prime to 5 we are assisted by the observation

$$
\operatorname{ord}_{5}\left(\tau^{2}-\tau-1\right)=\operatorname{ord}_{5}(f(\tau))=\operatorname{ord}_{5}(g(\tau))=0 \text { or } 1 .
$$

Proof of Corollary 3. Let $\tau \in \mathbf{Q}$ with $\tau \neq 0$. Theorem 1 gives two estimates for rank $E_{\tau}(\mathbf{Q})$. Writing $[\mathcal{A}, \mathcal{B}]$ for the matrix $\left(\chi_{q}(p)\right)_{p \in \mathcal{A}, q \in \mathcal{B}}$, these are

$$
\begin{aligned}
& |\mathcal{P}|+|\mathcal{Q}|+|\mathcal{R}|-1-2 \operatorname{rank}[\mathcal{P}, \mathcal{Q} \cup \mathcal{R}] \\
& |\mathcal{P}|+|\mathcal{Q}|+|\mathcal{R}|-1-2 \operatorname{rank}[\mathcal{P} \cup \mathcal{Q}, \mathcal{R}] .
\end{aligned}
$$

By repeated use of Dirichlet's theorem on primes in arithmetic progression, we choose $\mathcal{Q}_{0}$ and $\mathcal{R}_{0}$ disjoint sets of primes $p \equiv 1(\bmod 5)$ with $\operatorname{rank}\left[\mathcal{Q}_{0}, \mathcal{R}_{0}\right]$ arbitrarily large. With one final application of Dirichlet we choose $\tau$ prime such that $\mathcal{Q} \supset \mathcal{Q}_{0}$ and $\mathcal{R} \supset \mathcal{R}_{0}$. Then $|\mathcal{P}|=1$ and

$$
\operatorname{rank}[\mathcal{P}, \mathcal{Q} \cup \mathcal{R}] \ll \operatorname{rank}[\mathcal{P} \cup \mathcal{Q}, \mathcal{R}]
$$

The difference in the estimates (32) now proves the corollary.

A closer inspection of the proof of Corollary 3 shows that we are forcing elements of order 5 in the Tate-Shafarevich group of the elliptic curves isogenous to $E_{\tau}$ rather than for $E_{\tau}$ itself.

\subsection{Relationship with a formula of Cassels}

We compare our Theorem 1 with a formula of Cassels. This section is based on similar calculations in [Lo, §7].

Let $\phi: E \rightarrow E^{\prime}$ and $\widehat{\phi}: E^{\prime} \rightarrow E$ be dual isogenies of elliptic curves over $\mathbf{Q}$. We recall a formula of Cassels [Ca2], relating the order of the Selmer group attached to $\phi$ to the order of the Selmer group attached to $\widehat{\phi}$.

$$
\frac{\# S^{(\phi)}(E / \mathbf{Q})}{\# S^{(\widehat{\phi})}\left(E^{\prime} / \mathbf{Q}\right)}=\frac{\# E(\mathbf{Q})[\phi]}{\# E^{\prime}(\mathbf{Q})[\widehat{\phi}]} \times \frac{\Omega_{E^{\prime}}}{\Omega_{E}} \times \prod_{p} \frac{c_{p}\left(E^{\prime}\right)}{c_{p}(E)} .
$$

Here $\Omega_{E}=\int_{E(\mathbf{R})} \omega$ with $\omega$ the canonical Néron differential on $E$, and $c_{p}(E)=$ $\left[E\left(\mathbf{Q}_{p}\right): E_{0}\left(\mathbf{Q}_{p}\right)\right]$ is the Tamagawa number at the prime $p$. 
Let $n=5$ or 7 and let $\lambda \in \mathbf{Q}$ with $\lambda \neq 0$, respectively $\lambda \neq 0,1$. We check that (33), applied to the $n$-isogeny $\phi: C_{\lambda} \rightarrow D_{\lambda}$ is compatible with our descent calculations. By Theorem 1 the LHS of (33) is

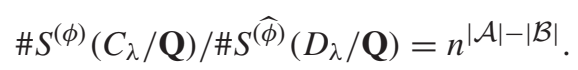

We now compute the RHS of (33). As seen in Sect. 1.1 we are always free to assume $\operatorname{ord}_{p}(\lambda) \geq 0$. The only primes $p$ to contribute to the product of Tamagawa numbers are the primes where $C_{\lambda}$ and $D_{\lambda}$ have split multiplicative reduction. For these primes the Weierstrass equations (2) and (3) are minimal and so $c_{p}=\operatorname{ord}_{p}(\Delta)$. By inspection of the discriminants (4) and (5) we find

$$
c_{p}\left(D_{\lambda}\right) / c_{p}\left(C_{\lambda}\right)= \begin{cases}n & \text { if } p \in \mathcal{A} \\ n^{-1} & \text { if } p \in \mathcal{B} .\end{cases}
$$

We write $\omega_{C}$ and $\omega_{D}$ for the Néron differentials on $C_{\lambda}$ and $D_{\lambda}$ respectively. Assuming $n \notin \mathcal{B}$, the Weierstrass equations (2) and (3) are both globally minimal. Since (3) was obtained from (2) using Vélu's formulae, it follows [V, Remarque 2] that $\omega_{D}=\widehat{\phi}^{*} \omega_{C}$. Thus

$$
\Omega_{D}=\int_{D_{\lambda}(\mathbf{R})} \omega_{D}=\int_{D_{\lambda}(\mathbf{R})} \widehat{\phi}^{*} \omega_{C}=\int_{\widehat{\phi} D_{\lambda}(\mathbf{R})} \omega_{C}=n \int_{C_{\lambda}(\mathbf{R})} \omega_{C}=n \Omega_{C}
$$

where $\widehat{\phi}: D_{\lambda}(\mathbf{R}) \rightarrow C_{\lambda}(\mathbf{R})$ is an $n$-fold cover. If $n \in \mathcal{B}$ then the Weierstrass equation (3) is no longer minimal at $p=n$. We then find $\omega_{D}=n^{-1} \widehat{\phi}^{*} \omega_{C}$ and $\Omega_{D}=\Omega_{C}$. Finally the RHS of (33) is

$$
\frac{1}{n} \times \frac{\Omega_{D}}{\Omega_{C}} \times \prod_{p} \frac{c_{p}\left(D_{\lambda}\right)}{c_{p}\left(C_{\lambda}\right)}=n^{|\mathcal{A}|-|\mathcal{B}|}
$$

and the formula is verified. This verification could equally be viewed as giving an alternative proof of the last part of Theorem 1.

\subsection{Tables of examples}

We apply Theorem 1 for some values $\lambda \in \mathbf{Q}$ chosen such that the elliptic curves $C_{\lambda}$ and $D_{\lambda}$ have conductor $N \leq 5300$. The list of curves considered is extracted from Cremona's tables [Cr3] by searching for curves with rational torsion of order 5, 7 or 10 . We recall that Cremona lists the curves by conductor, with a letter to distinguish isogeny classes with the same conductor, and a number to distinguish curves within each isogeny class. The quantities we tabulate are the conductor $N$, the Cremona labels \#, the sets of primes $\mathcal{A}$ and $\mathcal{B}$, the rank of the pairing $\Xi$, the dimensions of the Selmer groups $S^{(\phi)}\left(C_{\lambda} / \mathbf{Q}\right)$ and $S^{(\widehat{\phi})}\left(D_{\lambda} / \mathbf{Q}\right)$ and our estimate $r_{\phi}=|\mathcal{A}|+|\mathcal{B}|-1-2 \operatorname{rank}(\Xi)$ for the rank. Finally we compare with $r=\operatorname{rank} D_{\lambda}(\mathbf{Q})$ taken directly from Cremona's tables. Whenever the columns $r_{\phi}$ and $r$ differ, we deduce that there are elements of order $n$ in the Tate-Shafarevich group. We thus arrive at the lists of curves cited in the introduction. 
Table 1. $(\boldsymbol{n}=\mathbf{5})$ Descent via $\phi: C_{\lambda} \rightarrow D_{\lambda}$ over $\mathbf{Q}$

\begin{tabular}{|c|c|c|c|c|c|c|c|c|c|}
\hline$\lambda$ & $N$ & $\#$ & $\mathcal{A}$ & $\mathcal{B}$ & $\Xi$ & $\phi$ & $\widehat{\phi}$ & $r_{\phi}$ & $r$ \\
\hline 1 & 11 & $\mathrm{~A} 1,3$ & $\varnothing$ & $\{11\}$ & 0 & 0 & 1 & 0 & 0 \\
\hline 11 & 11 & $\mathrm{~A} 2,1$ & $\{11\}$ & $\emptyset$ & 0 & 1 & 0 & 0 & 0 \\
\hline 2 & 38 & $\mathrm{~B} 2,1$ & $\{2\}$ & $\emptyset$ & 0 & 1 & 0 & 0 & 0 \\
\hline-2 & 50 & B3, 1 & $\{2\}$ & $\emptyset$ & 0 & 1 & 0 & 0 & 0 \\
\hline 8 & 50 & B 4,2 & $\{2\}$ & $\varnothing$ & 0 & 1 & 0 & 0 & 0 \\
\hline 9 & 57 & $\mathrm{C} 2,1$ & $\{3\}$ & $\varnothing$ & 0 & 1 & 0 & 0 & 0 \\
\hline 4 & 58 & B2, 1 & $\{2\}$ & $\emptyset$ & 0 & 1 & 0 & 0 & 0 \\
\hline 12 & 66 & $\mathrm{C} 3,1$ & $\{2,3\}$ & $\{11\}$ & 1 & 1 & 0 & 0 & 0 \\
\hline $9 / 2$ & 66 & $\mathrm{C} 4,2$ & $\{2,3\}$ & $\{11\}$ & 1 & 1 & 0 & 0 & 0 \\
\hline 3 & 75 & $\mathrm{C} 2,1$ & $\{3\}$ & $\emptyset$ & 0 & 1 & 0 & 0 & 0 \\
\hline 10 & 110 & $\mathrm{~A} 2,1$ & $\{2,5\}$ & $\{11\}$ & 1 & 1 & 0 & 0 & 0 \\
\hline-4 & 118 & B2, 1 & $\{2\}$ & $\varnothing$ & 0 & 1 & 0 & 0 & 0 \\
\hline-3 & 123 & A 2,1 & $\{3\}$ & $\{41\}$ & 0 & 1 & 1 & 1 & 1 \\
\hline $4 / 3$ & 150 & $\mathrm{~A} 1,3$ & $\{2,3\}$ & $\{5\}$ & 1 & 1 & 0 & 0 & 0 \\
\hline 18 & 150 & $\mathrm{~A} 2,4$ & $\{2,3\}$ & $\{5\}$ & 1 & 1 & 0 & 0 & 0 \\
\hline 5 & 155 & $\mathrm{~A} 2,1$ & $\{5\}$ & $\{31\}$ & 0 & 1 & 1 & 1 & 1 \\
\hline 16 & 158 & $\mathrm{C} 2,1$ & $\{2\}$ & $\emptyset$ & 0 & 1 & 0 & 0 & 0 \\
\hline-7 & 175 & $\mathrm{~A} 1,2$ & $\{7\}$ & $\{5\}$ & 0 & 1 & 1 & 1 & 1 \\
\hline 6 & 186 & B2, 1 & $\{2,3\}$ & $\{31\}$ & 1 & 1 & 0 & 0 & 0 \\
\hline 7 & 203 & $\mathrm{~A} 2,1$ & $\{7\}$ & $\emptyset$ & 0 & 1 & 0 & 0 & 0 \\
\hline $32 / 3$ & 246 & $\mathrm{~B} 2,1$ & $\{2,3\}$ & $\{41\}$ & 1 & 1 & 0 & 0 & 0 \\
\hline $13 / 2$ & 286 & D2, 1 & $\{2,13\}$ & $\{11\}$ & 1 & 1 & 0 & 0 & 0 \\
\hline-8 & 302 & $\mathrm{~A} 2,1$ & $\{2\}$ & $\{151\}$ & 0 & 1 & 1 & 1 & 1 \\
\hline 13 & 325 & $\mathrm{E} 2,1$ & $\{13\}$ & $\varnothing$ & 0 & 1 & 0 & 0 & 0 \\
\hline $3 / 2$ & 366 & $\mathrm{~B} 2,1$ & $\{2,3\}$ & $\{61\}$ & 1 & 1 & 0 & 0 & 0 \\
\hline-5 & 395 & $\mathrm{C} 2,1$ & $\{5\}$ & $\varnothing$ & 0 & 1 & 0 & 0 & 0 \\
\hline$-3 / 2$ & 426 & A2, 1 & $\{2,3\}$ & $\{71\}$ & 1 & 1 & 0 & 0 & 0 \\
\hline-9 & 537 & $\mathrm{E} 2,1$ & $\{3\}$ & $\emptyset$ & 0 & 1 & 0 & 0 & 0 \\
\hline $11 / 2$ & 550 & $\mathrm{~K} 1,2$ & $\{2,11\}$ & $\{5\}$ & 1 & 1 & 0 & 0 & 0 \\
\hline-32 & 550 & $\mathrm{~K} 2,3$ & $\{2\}$ & $\{5,11\}$ & 1 & 0 & 1 & 0 & 0 \\
\hline $48 / 5$ & 570 & $\mathrm{~L} 3,1$ & $\{2,3,5\}$ & $\varnothing$ & 0 & 3 & 0 & 2 & 0 \\
\hline $100 / 9$ & 570 & $\mathrm{~L} 4,2$ & $\{2,3,5\}$ & $\varnothing$ & 0 & 3 & 0 & 2 & 0 \\
\hline 14 & 574 & $\mathrm{~J} 2,1$ & $\{2,7\}$ & $\{41\}$ & 1 & 1 & 0 & 0 & 0 \\
\hline-6 & 606 & $\mathrm{~F} 2,1$ & $\{2,3\}$ & $\{101\}$ & 1 & 1 & 0 & 0 & 0 \\
\hline $7 / 5$ & 665 & $\mathrm{D} 2,1$ & $\{5,7\}$ & $\emptyset$ & 0 & 2 & 0 & 1 & 1 \\
\hline $25 / 2$ & 710 & $\mathrm{D} 2,1$ & $\{2,5\}$ & $\{71\}$ & 1 & 1 & 0 & 0 & 0 \\
\hline $27 / 2$ & 786 & $\mathrm{M} 2,1$ & $\{2,3\}$ & $\{131\}$ & 1 & 1 & 0 & 0 & 0 \\
\hline-26 & 806 & $\mathrm{~F} 2,1$ & $\{2,13\}$ & $\{31\}$ & 1 & 1 & 0 & 0 & 0 \\
\hline$-4 / 3$ & 834 & $\mathrm{G} 2,1$ & $\{2,3\}$ & $\varnothing$ & 0 & 2 & 0 & 1 & 1 \\
\hline-16 & 862 & $\mathrm{E} 2,1$ & $\{2\}$ & $\{431\}$ & 0 & 1 & 1 & 1 & 1 \\
\hline $45 / 4$ & 870 & $\mathrm{I} 3,1$ & $\{2,3,5\}$ & $\varnothing$ & 0 & 3 & 0 & 2 & 0 \\
\hline $50 / 3$ & 870 & $\mathrm{I} 4,2$ & $\{2,3,5\}$ & $\varnothing$ & 0 & 3 & 0 & 2 & 0 \\
\hline $23 / 2$ & 874 & $\mathrm{E} 2,1$ & $\{2,23\}$ & $\emptyset$ & 0 & 2 & 0 & 1 & 1 \\
\hline
\end{tabular}




\begin{tabular}{|c|c|c|c|c|c|c|c|c|c|}
\hline$\lambda$ & $N$ & \# & $\mathcal{A}$ & $\mathcal{B}$ & $\Xi$ & $\phi$ & $\widehat{\phi}$ & $r_{\phi}$ & $r$ \\
\hline 15 & 885 & $\mathrm{D} 2,1$ & $\{3,5\}$ & $\emptyset$ & 0 & 2 & 0 & 1 & 1 \\
\hline $5 / 2$ & 890 & $\mathrm{G} 2,1$ & $\{2,5\}$ & $\varnothing$ & 0 & 2 & 0 & 1 & 1 \\
\hline $21 / 2$ & 1050 & $\mathrm{O} 2,1$ & $\{2,3,7\}$ & $\emptyset$ & 0 & 3 & 0 & 2 & 0 \\
\hline 37 & 1147 & $\mathrm{~B} 2,1$ & $\{37\}$ & $\{31\}$ & 0 & 1 & 1 & 1 & 1 \\
\hline$-15 / 7$ & 1155 & $\mathrm{~N} 2,1$ & $\{3,5,7\}$ & $\{11\}$ & 1 & 2 & 0 & 1 & 1 \\
\hline $8 / 3$ & 1254 & $\mathrm{~K} 2,1$ & $\{2,3\}$ & $\{11\}$ & 1 & 1 & 0 & 0 & 0 \\
\hline 27 & 1293 & $\mathrm{E} 2,1$ & $\{3\}$ & $\{431\}$ & 0 & 1 & 1 & 1 & 1 \\
\hline$-5 / 2$ & 1310 & $\mathrm{C} 2,1$ & $\{2,5\}$ & $\{131\}$ & 1 & 1 & 0 & 0 & 0 \\
\hline 32 & 1342 & $\mathrm{C} 2,1$ & $\{2\}$ & $\{11,61\}$ & 1 & 0 & 1 & 0 & 0 \\
\hline $122 / 11$ & 1342 & C3, 2 & $\{2,11,61\}$ & $\varnothing$ & 0 & 3 & 0 & 2 & 0 \\
\hline$-17 / 3$ & 1479 & $\mathrm{~F} 2,1$ & $\{3,17\}$ & $\emptyset$ & 0 & 2 & 0 & 1 & 1 \\
\hline $7 / 2$ & 1526 & $\mathrm{E} 2,1$ & $\{2,7\}$ & $\varnothing$ & 0 & 2 & 0 & 1 & 1 \\
\hline $32 / 13$ & 1586 & $\mathrm{D} 2,1$ & $\{2,13\}$ & $\{61\}$ & 1 & 1 & 0 & 0 & 0 \\
\hline$-9 / 2$ & 1650 & $\mathrm{R} 2,1$ & $\{2,3\}$ & $\{11\}$ & 1 & 1 & 0 & 0 & 0 \\
\hline-12 & 1650 & $\mathrm{~S} 2,1$ & $\{2,3\}$ & $\{11\}$ & 1 & 1 & 0 & 0 & 0 \\
\hline $16 / 3$ & 1686 & $\mathrm{C} 2,1$ & $\{2,3\}$ & $\{281\}$ & 1 & 1 & 0 & 0 & 0 \\
\hline 17 & 1717 & $\mathrm{C} 2,1$ & $\{17\}$ & $\{101\}$ & 0 & 1 & 1 & 1 & 1 \\
\hline 25 & 1745 & $\mathrm{E} 2,1$ & $\{5\}$ & $\varnothing$ & 0 & 1 & 0 & 0 & 0 \\
\hline 20 & 1790 & $\mathrm{D} 2,1$ & $\{2,5\}$ & $\emptyset$ & 0 & 2 & 0 & 1 & 1 \\
\hline 24 & 1866 & $\mathrm{I} 2,1$ & $\{2,3\}$ & $\{311\}$ & 1 & 1 & 0 & 0 & 0 \\
\hline$-85 / 8$ & 1870 & $\mathrm{H} 2,1$ & $\{2,5,17\}$ & $\{11\}$ & 1 & 2 & 0 & 1 & 1 \\
\hline$-8 / 3$ & 1914 & $\mathrm{O} 2,1$ & $\{2,3\}$ & $\{11\}$ & 1 & 1 & 0 & 0 & 0 \\
\hline-54 & 1914 & $\mathrm{P} 2,1$ & $\{2,3\}$ & $\{11\}$ & 1 & 1 & 0 & 0 & 0 \\
\hline$-68 / 3$ & 1938 & $\mathrm{~J} 2,1$ & $\{2,3,17\}$ & $\emptyset$ & 0 & 3 & 0 & 2 & 0 \\
\hline $144 / 13$ & 1950 & $\mathrm{Y} 2,1$ & $\{2,3,13\}$ & $\emptyset$ & 0 & 3 & 0 & 2 & 0 \\
\hline $9 / 4$ & 1986 & $\mathrm{G} 2,1$ & $\{2,3\}$ & $\{331\}$ & 1 & 1 & 0 & 0 & 0 \\
\hline-10 & 2090 & $\mathrm{~N} 2,1$ & $\{2,5\}$ & $\{11\}$ & 1 & 1 & 0 & 0 & 0 \\
\hline $5 / 4$ & 2110 & E2, 1 & $\{2,5\}$ & $\{211\}$ & 1 & 1 & 0 & 0 & 0 \\
\hline $56 / 5$ & 2170 & $\mathrm{Q} 2,1$ & $\{2,5,7\}$ & $\{31\}$ & 1 & 2 & 0 & 1 & 1 \\
\hline $29 / 3$ & 2175 & $\mathrm{~J} 1,2$ & $\{3,29\}$ & $\{5\}$ & 1 & 1 & 0 & 0 & 0 \\
\hline $5 / 3$ & 2235 & $\mathrm{~F} 2,1$ & $\{3,5\}$ & $\varnothing$ & 0 & 2 & 0 & 1 & 1 \\
\hline$-5 / 4$ & 2290 & $\mathrm{D} 2,1$ & $\{2,5\}$ & $\varnothing$ & 0 & 2 & 0 & 1 & 1 \\
\hline $19 / 2$ & 2318 & $\mathrm{E} 2,1$ & $\{2,19\}$ & $\{61\}$ & 1 & 1 & 0 & 0 & 0 \\
\hline $47 / 4$ & 2350 & N1, 2 & $\{2,47\}$ & $\{5\}$ & 1 & 1 & 0 & 0 & 0 \\
\hline $54 / 5$ & 2370 & $\mathrm{M} 2,1$ & $\{2,3,5\}$ & $\varnothing$ & 0 & 3 & 0 & 2 & 0 \\
\hline $34 / 3$ & 2550 & $\mathrm{EE} 2,1$ & $\{2,3,17\}$ & $\varnothing$ & 0 & 3 & 0 & 2 & 0 \\
\hline-11 & 2651 & $\mathrm{C} 2,1$ & $\{11\}$ & $\{241\}$ & 0 & 1 & 1 & 1 & 1 \\
\hline$-5 / 3$ & 2715 & $\mathrm{C} 2,1$ & $\{3,5\}$ & $\{181\}$ & 1 & 1 & 0 & 0 & 0 \\
\hline$-9 / 4$ & 2766 & $\mathrm{I} 2,1$ & $\{2,3\}$ & $\{461\}$ & 1 & 1 & 0 & 0 & 0 \\
\hline$-7 / 2$ & 2786 & $\mathrm{D} 2,1$ & $\{2,7\}$ & $\varnothing$ & 0 & 2 & 0 & 1 & 1 \\
\hline $27 / 4$ & 2850 & $\mathrm{~W} 2,1$ & $\{2,3\}$ & $\varnothing$ & 0 & 2 & 0 & 1 & 1 \\
\hline 19 & 2869 & $\mathrm{~B} 2,1$ & $\{19\}$ & $\{151\}$ & 0 & 1 & 1 & 1 & 1 \\
\hline $17 / 2$ & 3026 & $\mathrm{D} 2,1$ & $\{2,17\}$ & $\varnothing$ & 0 & 2 & 0 & 1 & 1 \\
\hline-27 & 3075 & $\mathrm{~L} 2,1$ & $\{3\}$ & $\{41\}$ & 0 & 1 & 1 & 1 & 1 \\
\hline
\end{tabular}




\begin{tabular}{|rccccccccc|}
\hline \multicolumn{1}{|c}{$\lambda$} & $N$ & $\#$ & $\mathcal{A}$ & $\mathcal{B}$ & $\Xi$ & $\phi$ & $\widehat{\phi}$ & $r_{\phi}$ & $r$ \\
\hline-18 & 3126 & $\mathrm{C} 2,1$ & $\{2,3\}$ & $\{521\}$ & 1 & 1 & 0 & 0 & 0 \\
$25 / 3$ & 3135 & $\mathrm{H} 2,1$ & $\{3,5\}$ & $\{11\}$ & 1 & 1 & 0 & 0 & 0 \\
$49 / 4$ & 3206 & $\mathrm{E} 2,1$ & $\{2,7\}$ & $\emptyset$ & 0 & 2 & 0 & 1 & 1 \\
$15 / 2$ & 3270 & $\mathrm{H} 2,1$ & $\{2,3,5\}$ & $\emptyset$ & 0 & 3 & 0 & 2 & 0 \\
$101 / 9$ & 3333 & $\mathrm{G} 2,1$ & $\{3,101\}$ & $\{11\}$ & 1 & 1 & 0 & 0 & 0 \\
$92 / 7$ & 3542 & $\mathrm{R} 2,1$ & $\{2,7,23\}$ & $\{11\}$ & 1 & 2 & 0 & 1 & 1 \\
$81 / 8$ & 3786 & $\mathrm{G} 2,1$ & $\{2,3\}$ & $\{631\}$ & 1 & 1 & 0 & 0 & 0 \\
$173 / 8$ & 3806 & $\mathrm{~K} 2,1$ & $\{2,173\}$ & $\{11\}$ & 1 & 1 & 0 & 0 & 0 \\
$7 / 4$ & 3850 & $\mathrm{~T} 2,1$ & $\{2,7\}$ & $\{11\}$ & 1 & 1 & 0 & 0 & 0 \\
$8 / 5$ & 4010 & $\mathrm{E} 2,1$ & $\{2,5\}$ & $\{401\}$ & 1 & 1 & 0 & 0 & 0 \\
$7 / 3$ & 4011 & $\mathrm{D} 2,1$ & $\{3,7\}$ & $\{191\}$ & 1 & 1 & 0 & 0 & 0 \\
-13 & 4043 & $\mathrm{~A} 2,1$ & $\{13\}$ & $\{311\}$ & 0 & 1 & 1 & 1 & 1 \\
21 & 4389 & $\mathrm{~K} 2,1$ & $\{3,7\}$ & $\{11\}$ & 1 & 1 & 0 & 0 & 0 \\
$89 / 8$ & 4450 & $\mathrm{~K} 2,1$ & $\{2,89\}$ & $\varnothing$ & 0 & 2 & 0 & 1 & 1 \\
-25 & 4495 & $\mathrm{D} 2,1$ & $\{5\}$ & $\{31\}$ & 0 & 1 & 1 & 1 & 1 \\
$-16 / 3$ & 4650 & $\mathrm{LL} 2,1$ & $\{2,3\}$ & $\{31\}$ & 1 & 1 & 0 & 0 & 0 \\
$9 / 8$ & 4650 & $\mathrm{PP} 2,1$ & $\{2,3\}$ & $\{31\}$ & 1 & 1 & 0 & 0 & 0 \\
-56 & 4774 & $\mathrm{~J} 2,1$ & $\{2,7\}$ & $\{11,31\}$ & 1 & 1 & 1 & 1 & 1 \\
$-7 / 4$ & 4774 & $\mathrm{~K} 2,1$ & $\{2,7\}$ & $\{11,31\}$ & 1 & 1 & 1 & 1 & 1 \\
$-8 / 5$ & 4790 & $\mathrm{C} 2,1$ & $\{2,5\}$ & $\emptyset$ & 0 & 2 & 0 & 1 & 1 \\
$-9 / 8$ & 4854 & $\mathrm{C} 2,1$ & $\{2,3\}$ & $\emptyset$ & 0 & 2 & 0 & 1 & 1 \\
-14 & 4886 & $\mathrm{~F} 2,1$ & $\{2,7\}$ & $\emptyset$ & 0 & 2 & 0 & 1 & 1 \\
$25 / 4$ & 4910 & $\mathrm{G} 2,1$ & $\{2,5\}$ & $\{491\}$ & 1 & 1 & 0 & 0 & 0 \\
-24 & 5034 & $\mathrm{E} 2,1$ & $\{2,3\}$ & $\varnothing$ & 0 & 2 & 0 & 1 & 1 \\
$43 / 4$ & 5074 & $\mathrm{D} 2,1$ & $\{2,43\}$ & $\varnothing$ & 0 & 2 & 0 & 1 & 1 \\
\hline & & & & & & & & &
\end{tabular}

Table 2. $(\boldsymbol{n}=7)$ Descent via $\phi: C_{\lambda} \rightarrow D_{\lambda}$ over $\mathbf{Q}$

\begin{tabular}{|rccccccccc|}
\hline \multicolumn{1}{r}{$\lambda$} & $N$ & $\#$ & $\mathcal{A}$ & $\mathcal{B}$ & $\Xi$ & $\phi$ & $\widehat{\phi}$ & $r_{\phi}$ & $r$ \\
\hline 2 & 26 & $\mathrm{~B} 2,1$ & $\{2\}$ & $\emptyset$ & 0 & 1 & 0 & 0 & 0 \\
3 & 174 & $\mathrm{~B} 2,1$ & $\{2,3\}$ & $\{29\}$ & 1 & 1 & 0 & 0 & 0 \\
4 & 258 & $\mathrm{~F} 2,1$ & $\{2,3\}$ & $\{43\}$ & 1 & 1 & 0 & 0 & 0 \\
-2 & 294 & $\mathrm{~B} 1,2$ & $\{2,3\}$ & $\{7\}$ & 1 & 1 & 0 & 0 & 0 \\
5 & 490 & $\mathrm{~K} 1,2$ & $\{2,5\}$ & $\{7\}$ & 1 & 1 & 0 & 0 & 0 \\
7 & 546 & $\mathrm{~F} 2,1$ & $\{2,3,7\}$ & $\varnothing$ & 0 & 3 & 0 & 2 & 0 \\
8 & 574 & $\mathrm{I} 2,1$ & $\{2,7\}$ & $\emptyset$ & 0 & 2 & 0 & 1 & 1 \\
-3 & 678 & $\mathrm{D} 2,1$ & $\{2,3\}$ & $\{113\}$ & 1 & 1 & 0 & 0 & 0 \\
9 & 762 & $\mathrm{G} 2,1$ & $\{2,3\}$ & $\{127\}$ & 1 & 1 & 0 & 0 & 0 \\
$-9 / 2$ & 858 & $\mathrm{~K} 2,1$ & $\{2,3,11\}$ & $\varnothing$ & 0 & 3 & 0 & 2 & 0 \\
6 & 1230 & $\mathrm{~K} 2,1$ & $\{2,3,5\}$ & $\varnothing$ & 0 & 3 & 0 & 2 & 0 \\
-4 & 2110 & $\mathrm{H} 2,1$ & $\{2,5\}$ & $\{211\}$ & 1 & 1 & 0 & 0 & 0 \\
-10 & 4730 & $\mathrm{~K} 2,1$ & $\{2,5,11\}$ & $\{43\}$ & 1 & 2 & 0 & 1 & 1 \\
$5 / 2$ & 5010 & $\mathrm{H} 2,1$ & $\{2,3,5\}$ & $\emptyset$ & 0 & 3 & 0 & 2 & 0 \\
\hline
\end{tabular}


Acknowledgements. The author would like to thank his supervisor Nick Shepherd-Barron for his guidance in researching this topic; John Coates, John Cremona, Cathy O'Neil and Ed Schaefer for comments and suggestions along the way; and John Cremona in particular for the use of his computer program mwrank. Nearly all of this article is taken directly from the author's $\mathrm{PhD}$ thesis.

\section{References}

[Bö] R. Bölling: Die Ordnung der Schafarewitsch-Tate-Gruppe kann beliebig gross werden. Math. Nachr. 67, 157-179 (1975)

[Ca1] J.W.S. Cassels: Arithmetic on curves of genus 1, VI. The Tate-Shafarevich group can be arbitrarily large. J. Reine Angew. Math. 214/215, 65-70 (1964)

[Ca2] J.W.S. Cassels: Arithmetic on curves of genus 1, VIII. On conjectures of Birch and Swinnerton-Dyer. J. Reine Angew. Math. 217, 180-199 (1965)

[Ca3] J.W.S. Cassels: Lectures on elliptic curves. LMSST 24: Cambridge University Press 1991

[CF] J.W.S. Cassels, A. Fröhlich (eds.): Algebraic number theory. Academic Press 1967

[Co] I. Connell: Calculating root numbers of elliptic curves over Q. Manuscripta Math. 82, 93-104 (1994)

[CM] J.E. Cremona, B. Mazur: Visualizing elements in the Shafarevich-Tate group. Exp. Math. 9(1), 13-28 (2000)

[Cr1] J.E. Cremona: Algorithms for modular elliptic curves (second edition). Cambridge University Press 1997

[Cr2] J.E. Cremona: mwrank, a program for 2-descent on elliptic curves over Q. See http: / / www. maths.nottingham.ac.uk/personal/jec/ftp/progs

[Cr3] J.E. Cremona: Modular elliptic curve data for conductors up to 5300. See http: / / www maths. nottingham.ac.uk/personal / jec/ftp/data

[F] T.A. Fisher: On 5 and 7 descents for elliptic curves. Cambridge PhD Thesis (2000)

[HR] H. Halberstam, H.-E. Richert: Sieve methods. LMS Monographs 4: Academic Press 1974

[H] K. Hulek: Projective geometry of elliptic curves. Soc. Math. de France, Astérisque 137 (1986)

[K1] F. Klein: Über die Transformation siebenter Ordnung der elliptischen Funktionen. Math. Ann. 14, 428-471 (1879). English translation in [Le] 287-331

[Kr] K. Kramer: A family of semistable elliptic curves with large Tate-Shafarevich groups. Proc. Am. Math. Soc. 89, 379-386 (1983)

[Le] S. Levy (ed.): The eightfold way, the beauty of Klein's quartic curve. MSRI Publications 35, Cambridge University Press 1999

[Lo] M. DeLong: A formula for the selmer group of a rational three-isogeny (1999)

[Ma1] B. Mazur: Arithmetic on curves. Bull. Am. Math. Soc. 14, 207-259 (1986)

[Ma2] B. Mazur: On the passage from local to global in number theory. Bull. Am. Math. Soc. 29, 14-50 (1993)

[Mi] J.S. Milne: Arithmetic duality theorems. Persp. in Math. 1. Academic Press 1986

[Mo] P. Monsky: Generalizing the Birch-Stephens theorem. Math. Z. 221, 415-420 (1996)

[Mu1] D. Mumford: On the equations defining abelian varieties. I. Invent. math. 1, 287-354 (1966)

[Mu2] D. Mumford: Abelian varieties. TIFR Studies in Math. 5: Oxford University Press 1970

[O'N] C.H. O'Neil: Jacobians of curves of genus one. Harvard PhD thesis (1999)

[R] D.E. Rohrlich: Variation of the root number in families of elliptic curves. Compositio Math. 87, 119-151 (1993)

[Se1] J.-P. Serre: Propriétés galoisiennes des points d'ordre fini des courbes elliptiques. Invent. math. 15, 259-331 (1972)

[Se2] J.-P. Serre: Local fields. GTM 67. Springer 1979 
[Si1] J.H. Silverman: The arithmetic of elliptic curves. GTM 106. Springer 1986

[Si2] J.H. Silverman: Advanced topics in the arithmetic of elliptic curves. GTM 151. Springer 1994

[T1] J. Tate: Duality theorems in Galois cohomology over number fields. Proc. Intern. Congress Math. Stockholm (1962), 288-295

[T2] J. Tate: The arithmetic of elliptic curves. Invent. math. 23, 179-206 (1974)

[V] J. Vélu: Isogénies entre courbes elliptiques. C. R. Acad. Sci. Paris 273, 238-241 (1971) 